\title{
AN UNCONDITIONALLY STABLE DISCONTINUOUS GALERKIN METHOD FOR THE ELASTIC HELMHOLTZ EQUATIONS WITH LARGE FREQUENCY
}

\author{
XIAOBING FENG* AND CODY LORTON ${ }^{\dagger}$
}

\begin{abstract}
In this paper we propose and analyze an interior penalty discontinuous Galerkin (IP-DG) method using piecewise linear polynomials for the elastic Helmholtz equations with the first order absorbing boundary condition. It is proved that the sesquilinear form for the problem satisfies a generalized weak coercivity property, which immediately infers a stability estimate for the solution of the differential problem in all frequency regimes. It is also proved that the proposed IPDG method is unconditionally stable with respect to both frequency $\omega$ and mesh size $h$. Sub-optimal order (with respect to $h$ ) error estimates in the broken $H^{1}$-norm and in the $L^{2}$-norm are obtained in all mesh regimes. These estimate improve to optimal order when the mesh size $h$ is restricted to the pre-asymptotic regime (i.e., $\omega^{\beta} h=O(1)$ for some $1 \leq \beta<2$ ). The novelties of the proposed IPDG method include: first, the method penalizes not only the jumps of the function values across the element edges but also the jumps of the normal derivatives; second, the penalty parameters are taken as complex numbers with positive imaginary parts. In order to establish the desired unconditional stability estimate for the numerical solution, the main idea is to exploit a (simple) property of linear functions to overcome the main difficulty caused by non-Hermitian nature and strong indefiniteness of the Helmholtz-type problem. The error estimate is then derived using a nonstandard technique adapted from [9]. Numerical experiments are also presented to validate the theoretical results and to numerically examine the pollution effect (with respect to $\omega$ ) in the error bounds.
\end{abstract}

Key words. Elastic Helmholtz equations, Korn's inequality, unconditional stability, discontinuous Galerkin methods, generalized weak coercivity, error estimates.

AMS subject classifications. $65 \mathrm{~N} 12,65 \mathrm{~N} 15,65 \mathrm{~N} 30,78 \mathrm{~A} 40$

1. Introduction. Wave phenomena are pervasive throughout many scientific fields, wave computation has been one of the central topics in computational sciences and has also been at the forefront of scientific computing. It becomes more and more important as the boundary of wave-related application problems keeps pushing outward. Solving these application problems largely hinges on computing the solutions of the governing wave equation(s). Among them those involving high frequency waves are often most difficult to analyze and solve numerically. This is due to the fact that a high frequency (or large wave number), which means a very short wave length, results in a strongly indefinite PDE problem and a highly oscillatory solution. Consequently, very fine meshes must be used to resolve the wave, which in turn results in extremely large non-Hermitian and strongly indefinite algebraic systems to solve, a daunting task especially in high dimensions.

In this paper we shall focus our attention on one type of wave phenomena, that is wave propagation through some isotropic elastic media. This type is often encountered in applications from materials science, medical science, petroleum engineering, just to name a few. Specifically, we shall consider the following elastic Helmholtz problem:

$$
\begin{array}{rlrl}
-\omega^{2} \rho \mathbf{u}-\operatorname{div}(\sigma(\mathbf{u})) & =\mathbf{f} & & \text { in } \Omega, \\
\mathbf{i} \omega A \mathbf{u}+\sigma(\mathbf{u}) \mathbf{n}=\mathbf{g} & & \text { on } \Gamma:=\partial \Omega,
\end{array}
$$

${ }^{*}$ Department of Mathematics, The University of Tennessee, Knoxville, TN 37996, U.S.A. (xfeng@math.utk.edu). The work of this author was partially supported by the NSF grants DMS1016173 and DMS-1318486.

${ }^{\dagger}$ Department of Mathematics and Statistics, University of West Florida, Pensacola, FL 32514, U.S.A. (clorton@uwf.edu). The work of this author was partially supported by the NSF grants DMS-1016173 and DMS-1318486. 
where $\Omega \subset \mathbb{R}^{d}, d=2,3$, which represents an isotropic elastic media, is an open and bounded domain. $\mathbf{n}$ denotes the unit outward normal vector to $\partial \Omega$, and $\mathbf{i}=$ $\sqrt{-1}$ denotes the imaginary unit. $\mathbf{u}: \Omega \rightarrow \mathbb{C}^{d}$ denotes the (Fourier-transformed) displacement vector of the elastic media $\Omega$. $\omega$ and $\rho$ are both positive constants for which $\omega$ denotes the frequency of the sought-after elastic wave traveling through $\Omega$ with speed $\rho$. $A$ is a $d \times d$ real symmetric positive definite matrix. $\sigma(\mathbf{u})$ is the stress-tensor in $\Omega$ defined by

$$
\sigma(\mathbf{u}):=2 \mu \varepsilon(\mathbf{u})+\lambda \operatorname{div} \mathbf{u} I, \quad \varepsilon(\mathbf{u}):=\frac{1}{2}\left(\nabla \mathbf{u}+\nabla \mathbf{u}^{T}\right)
$$

where $\mu>0$ and $\lambda>0$ are the Lamé constants for the elastic media $\Omega$.

Equations $(1.1)$ and $(1.2)$ can arise either from the frequency domain treatment of linear elastic wave equations (cf. [7]) or from seeking a time-harmonic solution to the following linear elastic wave equations:

$$
\begin{array}{rlrl}
\rho \mathbf{U}_{t t}-\operatorname{div}(\sigma(\mathbf{U})) & =\mathbf{F} & & \text { in } \Omega \times(0, \infty), \\
A \mathbf{U}+\sigma(\mathbf{U}) \mathbf{n}=\mathbf{G} & & \text { on } \Gamma \times(0, \infty), \\
\mathbf{U}=\mathbf{U}_{t} & =\mathbf{0} & & \text { in } \Omega \times\{t=0\}
\end{array}
$$

When $\mathbf{G}=\mathbf{0}$ the boundary condition 1.4 is known as the first-order absorbing (radiation) boundary condition [8]. This boundary condition is an artificial boundary condition which has the property of completely absorbing incoming plane waves that are perpendicular to the boundary $\partial \Omega$. This boundary condition is imposed on the boundary $\partial \Omega$ of the truncated domain $\Omega$ of some unbounded domain with the intent of making the problem computationally feasible.

As is the case for the scalar Helmholtz equation, which governs time-harmonic acoustic waves, the frequency $\omega$ also plays a key role in the analysis and implementation of any numerical method for the elastic Helmholtz equations. It is a well-known fact that in order to resolve the wave numerically one must use some minimum number of grid points in each wave length $\ell=2 \pi / \omega$ in every coordinate direction. This requires a minimum mesh constraint $\omega h=O(1)$, where $h$ denotes the mesh size. In fact, the widely held "rule of thumb" is to use $6-10$ grid points per wave length. In [12, Babǔska et al proved the necessity of this "rule of thumb" in the 1-dimensional case of the scalar Helmholtz equation. In [12] it was also shown that the $\mathrm{H}^{1}$ error bound for the finite element solution contains a pollution term that contributes to the loss of stability for the finite element method in the case of a large frequency. This pollution term also causes the error to increase as $\omega$ increases under the mesh constraint $\omega h=O(1)$. This forces one to adopt a more stringent mesh condition to guarantee an accurate numerical solution.

The loss of stability of the standard finite element method applied to Helmholtztype problems remains as an open issue to be resolved. In [1, 6, 7] a stringent mesh condition of $\omega^{2} h=O(1)$ (called the asymptotic mesh regime) was required to obtain optimal and quasi-optimal error estimates for the scalar Helmholtz equation. In [4] a similar mesh constraint of the form $\omega^{\beta} h=O(1)$ was used to obtain error estimates for the elastic Helmholtz equations, where $\beta \geq 2$ is a constant to be discussed later in this paper. Requiring such a stringent mesh constraint makes the use of a practical coarse approximation space impossible in the case that $\omega$ is large. This is a major hurdle that must be overcome if one wishes to use multi-level algebraic solvers such as the multigrid method or the multi-level domain decomposition method. 
The primary goal of this paper is to develop and analyze an interior penalty discontinuous Galerkin approximation method which is unconditionally stable with respect to both $\omega$ and $h$ for the above elastic Helmholtz problem, which is different from the goal of the earlier DG work 14. Specifically, we seek a numerical method in which a priori solution (or stability ) estimates can be obtained for all $\omega, h>0$. Our work in this paper can be regarded as the elastic counterpart of those reported in 9, 16, [1], where the scalar Helmholtz equation and the time-harmonic Maxwell's equations were the focuses of study. We like to note that all three Helmholtz-type problems share the above mentioned two main difficulties, namely, strong indefiniteness and non-Hermitian character. However, due to the fact that to their leading operators are very different, in particular, they have quite different kernel spaces, the formulation and analysis of the desired IP-DG methods are significantly different, although they are conceptually similar.

The remainder of the paper is organized as follows. In Section 2 we establish a new generalized weak coercivity property for the elastic operator $-\operatorname{div}(\sigma(\cdot))$, and derive a stability estimate for the PDE solution as a corollary of the generalized weak coercivity property. In Section 3 we introduce some notation and present the formulation of our IP-DG method studied in the rest of the paper. In Section 4 we derive a stability (or a priori solution) estimate for the numerical solution in all mesh regimes including the pre-asymptotic regime (i.e. $\omega^{\beta} h=O(1)$ for some $1 \leq \beta<2$ ). In Section 5 we employ the non-standard error estimate technique, which uses an elliptic projection along with the (discrete) stability estimate, to establish optimal order error estimates in both broken $\mathrm{H}^{1}$-norm and the $L^{2}$-norm. Section 6 is devoted to numerical experiments that validate our theoretical results and demonstrate the proposed method's advantage over the standard finite element method.

2. Generalized inf-sup condition and stability estimates for PDE solutions. The goal of this section is to prove that the sesquilinear form $a(\cdot, \cdot)$ defined in the weak formulation of 1.1)-(1.2) satisfies a generalized weak coercivity property. Similar results were also obtained for the scalar Helmholtz equation and the time-harmonic Maxwell's equations [11, 13. As a direct consequence of the weak coercivity property proved in this section, we will obtain stability estimates for the solution $\mathbf{u}$ of (1.1)-(1.2).

Throughout the rest of this paper we adopt the standard $L^{2}$-space norm and inner product notation. In particular, for $Q \subset \Omega$ and $\Sigma \subset \Gamma$, let $(\cdot, \cdot)_{Q}$ and $\langle\cdot, \cdot\rangle_{\Sigma}$ denote the $L^{2}$ inner product on the complex-valued inner product spaces $\mathbf{L}^{2}(Q)=\left(L^{2}(Q)\right)^{d}$ and $\mathbf{L}^{2}(\Sigma)=\left(L^{2}(\Sigma)\right)^{d}$, respectively.

We will study the weak formulation of $1.1-1.2$ defined as seeking $\mathbf{u} \in \mathbf{H}^{1}(\Omega)$ such that

$$
a(\mathbf{u}, \mathbf{v})=(\mathbf{f}, \mathbf{v})_{\Omega}+\langle\mathbf{g}, \mathbf{v}\rangle_{\Gamma} \quad \forall \mathbf{v} \in \mathbf{H}^{1}(\Omega),
$$

where $a(\cdot, \cdot)$ is the sesquilinear form defined on $\mathbf{H}^{1}(\Omega) \times \mathbf{H}^{1}(\Omega)$ as

$$
a(\mathbf{u}, \mathbf{v}):=\lambda(\operatorname{div} \mathbf{u}, \operatorname{div} \mathbf{v})_{\Omega}+2 \mu(\varepsilon(\mathbf{u}), \varepsilon(\mathbf{v}))_{\Omega}-\omega^{2} \rho(\mathbf{u}, \mathbf{v})_{\Omega}+\mathbf{i} \omega\langle A \mathbf{u}, \mathbf{v}\rangle_{\Gamma} .
$$

To show that $a(\cdot, \cdot)$ satisfies a generalized weak coercivity property we will need to assume that the domain $\Omega$ is "nice". In particular, we assume that $\Omega$ is a strictly star-shaped domain, i.e. there exists $x_{0} \in \Omega$ and $c_{0}>0$ such that

$$
\left(\mathbf{x}-\mathbf{x}_{0}\right) \cdot \mathbf{n} \geq c_{0} \quad \forall x \in \Gamma .
$$


We will need to make use of Korn's second inequality to achieve our result. This inequality is given in the following lemma.

Lemma 2.1. There exists a positive constant $K$ such that for any $\mathbf{v} \in \mathbf{H}^{1}(\Omega)$ the following inequality holds

$$
\|\mathbf{v}\|_{H^{1}(\Omega)} \leq K\left[\|\varepsilon(\mathbf{v})\|_{L^{2}(\Omega)}+\|\mathbf{v}\|_{L^{2}(\Omega)}\right] .
$$

Lastly, we will need to assume that the solution $\mathbf{u}$ to 2.1) satisfies a Korn-type inequality on the boundary $\Gamma$. This boundary Korn inequality was conjectured in $[5$ and it was necessary for the authors to obtain stability estimates for the Elastic Helmholtz equation that are optimal in the frequency $\omega$. Here it is stated as a conjecture.

CONJECTURe 2.2. There exists a positive constant $\tilde{K}$ such that for any $\mathbf{u} \in$ $\mathbf{H}^{2}(\Omega)$, the following Korn-type inequality holds

$$
\|\boldsymbol{\nabla} \mathbf{u}\|_{L^{2}(\Gamma)}^{2} \leq \tilde{K}\left[\|\mathbf{u}\|_{L^{2}(\Gamma)}^{2}+\|\varepsilon(\mathbf{u})\|_{L^{2}(\Gamma)}^{2}\right] .
$$

With this boundary Korn inequality in mind, we define the following function spaces.

$$
\begin{aligned}
\mathbf{V} & :=\left\{\mathbf{v} \in \mathbf{H}^{1}(\Omega) ;|\nabla \mathbf{u}|_{\Gamma} \in \mathbf{L}^{2}(\Gamma)\right\}, \\
\mathbf{V}_{\tilde{K}} & :=\{\mathbf{v} \in \mathbf{V} ; 2.5 \text { holds with constant } \tilde{K}\} .
\end{aligned}
$$

We also note that since $A$ in $(1.2)$ is $\mathrm{SPD}$, there exist positive constants $c_{A}$ and $C_{A}$ such that

$$
c_{A}\|\mathbf{v}\|_{L^{2}(\Gamma)}^{2} \leq\langle A \mathbf{v}, \mathbf{v}\rangle_{\Gamma} \leq C_{A}\|\mathbf{v}\|_{L^{2}(\Gamma)}^{2} \quad \forall \mathbf{v} \in \mathbf{L}^{2}(\Gamma) .
$$

The following theorem states the generalized weak coercivity property for the sesquilinear form $a(\cdot, \cdot)$.

THEOREM 2.3. Let $\Omega$ be a strictly star-shaped domain with diam $(\Omega)=R$ and let $\tilde{K}$ be a positive constant. Then for any $\mathbf{u} \in \mathbf{V}_{\tilde{K}}$ the following inequality holds

$$
\sup _{\mathbf{v} \in \mathbf{V}} \frac{|\operatorname{Im} a(\mathbf{u}, \mathbf{v})|}{\|\mathbf{v}\|_{E}}+\sup _{\mathbf{v} \in \mathbf{H}^{1}(\Omega)} \frac{|\operatorname{Re} a(\mathbf{u}, \mathbf{v})|}{\|\mathbf{v} \mid\|_{L^{2}(\Omega)}} \geq \frac{1}{\gamma}\|\mathbf{u}\|_{E},
$$

where

$$
\begin{aligned}
\gamma:= & {\left[4 R^{2} K\left(1+\frac{\omega^{2} \rho}{2 \mu}\right)+4 R^{2} \tilde{K}+(d-1)^{2}\right]^{\frac{1}{2}} } \\
M:= & \frac{1}{c_{A} c_{0} \mu}\left(R \omega^{2} \rho c_{0} \mu+R^{2} \omega C_{A} \tilde{K}+c_{0}^{2} \mu^{2}\right) \\
\|\mathbf{v}\|_{E}^{2}:=\omega^{2} \rho\|\mathbf{v}\|_{L^{2}(\Omega)}^{2}+\lambda\|\operatorname{div} \mathbf{v}\|_{L^{2}(\Omega)}^{2}+2 \mu\|\varepsilon(\mathbf{v})\|_{L^{2}(\Omega)} & \quad+c_{0} \lambda\|\operatorname{div} \mathbf{v}\|_{L^{2}(\Gamma)}^{2}+c_{0} \mu\|\varepsilon(\mathbf{v})\|_{L^{2}(\Gamma)}^{2}, \\
\|\mathbf{v}\|_{L^{2}(\Omega)}^{2}:= & \omega^{2} \rho\|\mathbf{v}\|_{L^{2}(\Omega)}^{2}+c_{0} \mu\|\mathbf{v}\|_{L^{2}(\Gamma)}^{2} .
\end{aligned}
$$


Proof. In this proof we assume that $\mathbf{u} \in \mathbf{V}_{\tilde{K}} \cap \mathbf{H}^{2}(\Omega)$, noting that once we obtain the result on this more restrictive space that we can obtain the desired result for $\mathbf{u} \in \mathbf{V}_{\tilde{K}}$ by the standard density argument.

By letting $\mathbf{v}=\mathbf{u}$ in 2.2 and taking the real and imaginary parts separately we get

$$
\begin{aligned}
& \operatorname{Re} a(\mathbf{u}, \mathbf{u})=\lambda\|\operatorname{div} \mathbf{u}\|_{L^{2}(\Omega)}^{2}+2 \mu\|\varepsilon(\mathbf{u})\|_{L^{2}(\Omega)}^{2}-\omega^{2} \rho\|\mathbf{u}\|_{L^{2}(\Omega)}^{2}, \\
& \operatorname{Im} a(\mathbf{u}, \mathbf{u})=\omega\langle A \mathbf{u}, \mathbf{u}\rangle_{\Gamma^{\circ}}
\end{aligned}
$$

For the remainder of this proof we let $\mathbf{v}=(\boldsymbol{\nabla} \mathbf{u}) \boldsymbol{\alpha}$, where $\boldsymbol{\alpha}:=\mathbf{x}-\mathbf{x}_{0}$ and $\mathbf{x}_{0} \in \Omega$ comes from the star-shape property on the domain given by $(2.3)$. With this choice of test function $\mathbf{v}$, we can use the following integral identities for the elastic Helmholtz operator [5].

$$
\begin{aligned}
& \lambda\left\langle\boldsymbol{\alpha} \cdot \mathbf{n},|\operatorname{div} \mathbf{u}|^{2}\right\rangle_{\Gamma}+2 \mu\left\langle\boldsymbol{\alpha} \cdot \mathbf{n},|\varepsilon(\mathbf{u})|^{2}\right\rangle_{\Gamma} \\
& =(d-2)\left(\lambda\|\operatorname{div} \mathbf{u}\|_{L^{2}(\Omega)}^{2}+2 \mu\|\varepsilon(\mathbf{u})\|_{L^{2}(\Omega)}^{2}\right) \\
& \quad+2 \operatorname{Re}\left(\lambda(\operatorname{div} \mathbf{u}, \operatorname{div} \mathbf{v})_{\Omega}+2 \mu(\varepsilon(\mathbf{u}), \varepsilon(\mathbf{v}))_{\Omega}\right)
\end{aligned}
$$

and

$$
d\|\mathbf{u}\|_{L^{2}(\Omega)}^{2}=\left\langle\boldsymbol{\alpha} \cdot \mathbf{n},|\mathbf{u}|^{2}\right\rangle_{\Gamma}-2 \operatorname{Re}(\mathbf{u}, \mathbf{v})_{\Omega} .
$$

With $\mathbf{v}=(\boldsymbol{\nabla u}) \boldsymbol{\alpha}$ in 2.2 , we apply the above identities to get

$$
\begin{aligned}
& 2 \operatorname{Re} a(\mathbf{u}, \mathbf{v})=\lambda\left\langle\boldsymbol{\alpha} \cdot \mathbf{n},|\operatorname{div} \mathbf{u}|^{2}\right\rangle_{\Gamma}+2 \mu\left\langle\boldsymbol{\alpha} \cdot \mathbf{n},|\varepsilon(\mathbf{u})|^{2}\right\rangle_{\Gamma} \\
&-(d-2)\left(\lambda\|\operatorname{div} \mathbf{u}\|_{L^{2}(\Omega)}^{2}+2 \mu\|\varepsilon(\mathbf{u})\|_{L^{2}(\Omega)}^{2}\right) \\
&+d \omega^{2} \rho\|\mathbf{u}\|_{L^{2}(\Omega)}^{2}-\omega^{2} \rho\left\langle\boldsymbol{\alpha} \cdot \mathbf{n},|\mathbf{u}|^{2}\right\rangle_{\Gamma} \\
&-2 \omega \operatorname{Im}\langle A \mathbf{u}, \mathbf{v}\rangle_{\Gamma} .
\end{aligned}
$$

Rearranging the terms gives

$$
\begin{aligned}
d \omega^{2} \rho\|\mathbf{u}\|_{L^{2}(\Omega)}^{2}-(d-2)\left(\lambda\|\operatorname{div} \mathbf{u}\|_{L^{2}(\Omega)}^{2}+2 \mu\|\varepsilon(\mathbf{u})\|_{L^{2}(\Omega)}^{2}\right) & \\
=- & \lambda\left\langle\boldsymbol{\alpha} \cdot \mathbf{n},|\operatorname{div} \mathbf{u}|^{2}\right\rangle_{\Gamma}-2 \mu\left\langle\boldsymbol{\alpha} \cdot \mathbf{n},|\varepsilon(\mathbf{u})|^{2}\right\rangle_{\Gamma}+\omega^{2} \rho\left\langle\boldsymbol{\alpha} \cdot \mathbf{n},|\mathbf{u}|^{2}\right\rangle_{\Gamma} \\
& \quad+2 \omega \operatorname{Im}\langle A \mathbf{u}, \mathbf{v}\rangle_{\Gamma}+2 \operatorname{Re} a(\mathbf{u}, \mathbf{v}) .
\end{aligned}
$$

Adding $(2.12)$ and $(d-1)$ times 2.8$)$ yields

$$
\begin{aligned}
\omega^{2} \rho\|\mathbf{u}\|_{L^{2}(\Omega)}^{2}+\lambda\|\operatorname{div} \mathbf{u}\|_{L^{2}(\Omega)}^{2}+2 \mu\|\varepsilon(\mathbf{u})\|_{L^{2}(\Omega)}^{2} & \\
=- & \lambda\left\langle\boldsymbol{\alpha} \cdot \mathbf{n},|\operatorname{div} \mathbf{u}|^{2}\right\rangle_{\Gamma}-2 \mu\left\langle\boldsymbol{\alpha} \cdot \mathbf{n},|\varepsilon(\mathbf{u})|^{2}\right\rangle_{\Gamma}+\omega^{2} \rho\left\langle\boldsymbol{\alpha} \cdot \mathbf{n},|\mathbf{u}|^{2}\right\rangle_{\Gamma} \\
& \quad+2 \omega \operatorname{Im}\langle A \mathbf{u}, \mathbf{v}\rangle_{\Gamma}+\operatorname{Re} a(\mathbf{u},(d-1) \mathbf{u}+2 \mathbf{v}) .
\end{aligned}
$$

Now applying 2.3), 2.6), and Young's inequality yields

$$
\begin{aligned}
& \omega^{2} \rho\|\mathbf{u}\|_{L^{2}(\Omega)}^{2}+\lambda\|\operatorname{div} \mathbf{u}\|_{L^{2}(\Omega)}^{2}+2 \mu\|\varepsilon(\mathbf{u})\|_{L^{2}(\Omega)}^{2} \\
& \leq-c_{0}\left(\lambda\|\operatorname{div} \mathbf{u}\|_{L^{2}(\Gamma)}^{2}+2 \mu\|\varepsilon(\mathbf{u})\|_{L^{2}(\Gamma)}^{2}\right)+R \omega^{2} \rho\|\mathbf{u}\|_{L^{2}(\Gamma)}^{2} \\
& \quad+\frac{R^{2} \omega^{2} C_{A}}{\delta}\|\mathbf{u}\|_{L^{2}(\Gamma)}^{2}+\delta\|\nabla \mathbf{v}\|_{L^{2}(\Gamma)}^{2}+\operatorname{Re} a(\mathbf{u},(d-1) \mathbf{u}+2 \mathbf{v}) .
\end{aligned}
$$


Noting that $\mathbf{u} \in \mathbf{V}_{\tilde{K}}$ and choosing $\delta=\frac{c_{0} \mu}{\tilde{K}}$ yields

$$
\begin{aligned}
& \omega^{2} \rho\|\mathbf{u}\|_{L^{2}(\Omega)}^{2}+\lambda\|\operatorname{div} \mathbf{u}\|_{L^{2}(\Omega)}^{2}+2 \mu\|\varepsilon(\mathbf{u})\|_{L^{2}(\Omega)}^{2}+c_{0}\left(\lambda\|\operatorname{div} \mathbf{u}\|_{L^{2}(\Gamma)}^{2}+2 \mu\|\varepsilon(\mathbf{u})\|_{L^{2}(\Gamma)}^{2}\right) \\
& \quad \leq \frac{1}{\omega c_{A}}\left(R \omega^{2} \rho+\frac{R^{2} \omega^{2} C_{A} \tilde{K}}{c_{0} \mu}+c_{0} \mu\right) \omega c_{A}\|\mathbf{u}\|_{L^{2}(\Gamma)}^{2}+\operatorname{Re} a(\mathbf{u},(d-1) \mathbf{u}+2 \mathbf{v}) \\
& \quad \leq\left(\frac{R \omega^{2} \rho c_{0} \mu+R^{2} \omega C_{A} \tilde{K}+c_{0}^{2} \mu^{2}}{\omega c_{A} c_{0} \mu}\right) \omega\langle A \mathbf{u}, \mathbf{u}\rangle_{\Gamma}+\operatorname{Re} a(\mathbf{u},(d-1) \mathbf{u}+2 \mathbf{v}) \\
& \quad=|\operatorname{Re} a(\mathbf{u}, \hat{\mathbf{v}})|+M|\operatorname{Im} a(\mathbf{u}, \mathbf{u})| .
\end{aligned}
$$

where $\hat{\mathbf{v}}:=(d-1) \mathbf{u}+2 \mathbf{v}$. Thus

$$
\|\mathbf{u}\|_{E}^{2} \leq|\operatorname{Re} a(\mathbf{u}, \hat{\mathbf{v}})|+M|\operatorname{Im} a(\mathbf{u}, \mathbf{u})|
$$

By the definitions of $\|\cdot\|_{E}$ and $\||\cdot|\|_{L^{2}(\Omega)}$ and using (2.4) we obtain

$$
\begin{aligned}
\left.\|\hat{\mathbf{v}}\|\right|_{L^{2}(\Omega)} ^{2}= & \omega^{2} \rho\|\hat{\mathbf{v}}\|_{L^{2}(\Omega)}^{2}+c_{0} \mu\|\hat{\mathbf{v}}\|_{\Gamma}^{2} \\
\leq & 4 R^{2} \omega^{2} \rho\|\nabla \mathbf{u}\|_{L^{2}(\Omega)}^{2}+4 R^{2} c_{0} \mu\|\nabla \mathbf{u}\|_{\Gamma}^{2} \\
& \quad+(d-1)^{2} \omega^{2} \rho\|\mathbf{u}\|_{L^{2}(\Omega)}+(d-1)^{2} c_{0} \mu\|\mathbf{u}\|_{L^{2}(\Gamma)}^{2} \\
\leq & {\left[4 R^{2} K\left(1+\frac{\omega^{2} \rho}{2 \mu}\right)+4 R^{2} \tilde{K}+(d-1)^{2}\right]\|\mathbf{u}\|_{E}^{2} . }
\end{aligned}
$$

Thus

$$
\left.\|\hat{\mathbf{v}}\|\right|_{L^{2}(\Omega)} \leq \gamma\|\mathbf{u}\|_{E}
$$

It follows from 2.13 and 2.14 that

$$
\begin{aligned}
\sup _{\mathbf{v} \in \mathbf{V}} \frac{|\operatorname{Im} a(\mathbf{u}, \mathbf{v})|}{\|\mathbf{v}\|_{E}}+\sup _{\mathbf{v} \in \mathbf{H}^{1}(\Omega)} \frac{|\operatorname{Re} a(\mathbf{u}, \mathbf{v})|}{\|\mathbf{v} \mid\|_{L^{2}(\Omega)}} & \geq \frac{|\operatorname{Im} a(\mathbf{u}, \mathbf{u})|}{\|\mathbf{u}\|_{E}}+\frac{|\operatorname{Re} a(\mathbf{u}, \hat{\mathbf{v}})|}{\|\hat{\mathbf{v}}\| \|_{L^{2}(\Omega)}} \\
& \geq \frac{M|\operatorname{Im} a(\mathbf{u}, \mathbf{u})|+|\operatorname{Re} a(\mathbf{u}, \hat{\mathbf{v}})|}{\gamma\|\mathbf{u}\|_{E}} \\
& \geq \frac{1}{\gamma}\|\mathbf{u}\|_{E} .
\end{aligned}
$$

The proof is complete.

The generalized weak coercivity property of $a(\cdot, \cdot)$ given in Theorem 2.3 immediately infers the following stability estimate for the solution to (2.1).

THEOREM 2.4. Let $\Omega$ be a strictly star-shaped domain with $\operatorname{diam}(\Omega)=R$. Furthermore, suppose there exists some positive constant $\tilde{K}$ such that $\mathbf{u} \in \mathbf{V}_{\tilde{K}}$ solves (2.1) for $\mathbf{f} \in \mathbf{L}^{2}(\Omega)$ and $\mathbf{g} \in \mathbf{L}^{2}(\Gamma)$. Then the following stability result holds:

$$
\|\mathbf{u}\|_{E} \leq 2 \gamma\left(\frac{1}{\omega^{2} \rho}+\frac{1}{c_{0} \mu}\right)\left(\|\mathbf{f}\|_{L^{2}(\Omega)}+\|\mathbf{g}\|_{L^{2}(\Gamma)}\right),
$$

where $\|\cdot\|_{E}$ and $\gamma$ are the same as those defined in Theorem 2.3. 
Proof. By the Cauchy-Schwarz inequality, for any $\mathbf{v} \in \mathbf{H}^{1}(\Omega)$ the following result holds

$$
\begin{aligned}
|a(\mathbf{u}, \mathbf{v})| & =\mid(\mathbf{f}, \mathbf{v})+\langle\mathbf{g}, \mathbf{v}\langle\Gamma| \\
& \leq\|\mathbf{f}\|_{L^{2}(\Omega)}\|\mathbf{v}\|_{L^{2}(\Omega)}+\|\mathbf{g}\|_{L^{2}(\Gamma)}\|\mathbf{v}\|_{L^{2}(\Gamma)} \\
& \leq\left(\|\mathbf{f}\|_{L^{2}(\Omega)}^{2}+\|\mathbf{g}\|_{L^{2}(\Gamma)}^{2}\right)^{\frac{1}{2}}\left(\|\mathbf{v}\|_{L^{2}(\Omega)}^{2}+\|\mathbf{v}\|_{L^{2}(\Gamma)}^{2}\right)^{\frac{1}{2}} \\
& \leq\left(\frac{1}{\omega^{2} \rho}+\frac{1}{c_{0} \mu}\right)\|\mid \mathbf{v}\| \|_{L^{2}(\Omega)}\left(\|\mathbf{f}\|_{L^{2}(\Omega)}+\|\mathbf{g}\|_{L^{2}(\Gamma)}\right) \\
& \leq\left(\frac{1}{\omega^{2} \rho}+\frac{1}{c_{0} \mu}\right)\|\mathbf{v}\|_{E}\left(\|\mathbf{f}\|_{L^{2}(\Omega)}+\|\mathbf{g}\|_{L^{2}(\Gamma)}\right)
\end{aligned}
$$

Combining this inequality with the result of Theorem 2.3 yields

$$
\begin{aligned}
\frac{1}{\gamma}\|\mathbf{u}\|_{E} & \leq \sup _{\mathbf{v} \in \mathbf{V}} \frac{|\operatorname{Im} a(\mathbf{u}, \mathbf{v})|}{\|\mathbf{v}\|_{E}}+\sup _{\mathbf{v} \in \mathbf{H}^{1}(\Omega)} \frac{|\operatorname{Re} a(\mathbf{u}, \mathbf{v})|}{\|\mathbf{v}\|_{L^{2}(\Omega)}} \\
& \leq 2\left(\frac{1}{\omega^{2} \rho}+\frac{1}{c_{0} \mu}\right)\left(\|\mathbf{f}\|_{L^{2}(\Omega)}+\|\mathbf{g}\|_{L^{2}(\Gamma)}\right) .
\end{aligned}
$$

The proof is complete. $\square$

REMARK 2.5. We remark that both estimates (2.7) and 2.15) still hold without Conjecture 2.2. but the dependence of the upper bounds on $\omega$ would be slightly stronger as shown in [5].

3. Formulation of the IP-DG scheme. In this section we shall present our IP-DG scheme for the elastic Helmholtz equations. Conceptually, the formulation will mimic those for the scalar Helmholtz problem and the Maxwell problem given in [9, 10]. To do so we first need to introduce some notation.

Let $\mathcal{T}_{h}$ be a shape regular triangulation of the domain $\Omega$ such that for each triangle/tetrahedron $K \in \mathcal{T}_{h}, h \geq h_{K}:=\operatorname{diam}(K)$. Also for each edge/face $e$ of triangle $K$ define $h_{e}:=\operatorname{diam}(e)$. Next we define the following notation for the set of edges of $\mathcal{T}_{h}$ :

$$
\begin{aligned}
& \mathcal{E}_{h}^{I}:=\text { set of all interior edges/faces of } \mathcal{T}_{h}, \\
& \mathcal{E}_{h}^{B}:=\text { set of all boundary edges/faces of } \mathcal{T}_{h} \text { on } \Gamma .
\end{aligned}
$$

For each $e \in \mathcal{E}_{h}^{I}$ there exists $K_{e}, K_{e}^{\prime} \in \mathcal{T}_{h}$ such that $e=\partial K_{e} \cap \partial K_{e}^{\prime}$ and thus we define the jump and average operators on $e$ as follows:

$\left.[\mathbf{v}]\right|_{e}:= \begin{cases}\left.\mathbf{v}\right|_{K_{e}}-\left.\mathbf{v}\right|_{K_{e}^{\prime}}, & \text { if the global labeling number of } K_{e} \text { is greater than that of } K_{e}^{\prime}, \\ \left.\mathbf{v}\right|_{K_{e}^{\prime}}-\left.\mathbf{v}\right|_{K_{e}}, & \text { if the global labeling number of } K_{e}^{\prime} \text { is greater than that of } K_{e}\end{cases}$

and

$$
\left.\{\mathbf{v}\}\right|_{e}:=\frac{1}{2}\left(\left.\mathbf{v}\right|_{K_{e}}+\left.\mathbf{v}\right|_{K_{e}^{\prime}}\right) .
$$

Note that for $e \in \mathcal{E}_{h}^{B}$, we use the convention $\left.[\mathbf{v}]\right|_{e}=\left.\{\mathbf{v}\}\right|_{e}:=\left.\mathbf{v}\right|_{e}$. Also for $e \in \mathcal{E}_{h}^{I}$ we shall need to define the outward normal vector $\mathbf{n}_{e}$ to $e=\partial K_{e} \cap \partial K_{e}^{\prime}$. Let $\mathbf{n}_{e}$ be the unit outward normal vector to $K_{e}$ on $e$ if $K_{e}$ has a greater labeling number than $K_{e}^{\prime}$, and the unit outward normal vector to $K_{e}^{\prime}$ if the opposite is true. 
Next, we introduce the energy space $\mathbf{E}$ and the sesquilinear form $a_{h}(\cdot, \cdot)$ on $\mathbf{E} \times \mathbf{E}$ as follows:

$$
\begin{aligned}
& \mathbf{E}:=\prod_{K \in \mathcal{T}_{h}} \mathbf{H}^{2}(K):=\prod_{K \in \mathcal{T}_{h}}\left(H^{2}(K)\right)^{d} \\
& a_{h}(\mathbf{u}, \mathbf{v}):=b_{h}(\mathbf{u}, \mathbf{v})+\mathbf{i}\left(J_{0}(\mathbf{u}, \mathbf{v})+J_{1}(\mathbf{u}, \mathbf{v})\right) \quad \forall \mathbf{u}, \mathbf{v} \in \mathbf{E},
\end{aligned}
$$

where

$$
\begin{aligned}
b_{h}(\mathbf{u}, \mathbf{v}):= & \sum_{K \in \mathcal{T}_{h}}\left(\lambda(\operatorname{div} \mathbf{u}, \operatorname{div} \mathbf{v})_{K}+2 \mu(\varepsilon(\mathbf{u}), \varepsilon(\mathbf{v}))_{K}\right)-\sum_{e \in \mathcal{E}_{h}^{I}}\left\langle\left\{\sigma(\mathbf{u}) \mathbf{n}_{e}\right\},[\mathbf{v}]\right\rangle_{e} \\
& +\eta \sum_{e \in \mathcal{E}_{h}^{I}}\left\langle[\mathbf{u}],\left\{\sigma(\mathbf{v}) \mathbf{n}_{e}\right\}\right\rangle_{e}, \\
J_{0}(\mathbf{u}, \mathbf{v}):= & \sum_{e \in \mathcal{E}_{h}^{I}} \frac{\gamma_{0, e}}{h_{e}}\langle[\mathbf{u}],[\mathbf{v}]\rangle, \\
J_{1}(\mathbf{u}, \mathbf{v}):= & \sum_{e \in \mathcal{E}_{h}^{I}} \gamma_{1, e} h_{e}\left\langle\left[\sigma(\mathbf{u}) \mathbf{n}_{e}\right],\left[\sigma(\mathbf{v}) \mathbf{n}_{e}\right]\right\rangle .
\end{aligned}
$$

Here $\eta$ is an $h$-independent "symmetrization" parameter and $\gamma_{0, e}$, and $\gamma_{1, e}$ are nonnegative "penalty" parameters that will be specified later. For the rest of this paper we shall restrict ourselves to the case $\eta=-1$ (i.e. the symmetric case) but we note that other values of $\eta$ are possible.

REMARK 3.1. (a) Both $\mathbf{i} J_{0}$ and $\mathbf{i} J_{1}$ terms are called interior penalty terms. To the best our knowledge, penalizing the jumps of the normal stress $\sigma(\mathbf{u}) \mathbf{n}_{e}$ across the element interfaces seems have not been used before, and this term will play an important role in our stability and error analysis to be given in the next two sections.

(b) Another new feature of the sesquilinear form $a_{h}$ is that the penalty constants in $\mathbf{i} J_{0}$ and $\mathbf{i} J_{1}$ are complex numbers and must have positive imaginary parts. This feature will be vital for constructing our unconditionally stable IP-DG scheme.

(c) It is easy to see that $a_{h}(\cdot, \cdot)$ is a consistent discretization of the elastic operator $-\operatorname{div}(\sigma(\cdot))$.

We shall also define some appropriate semi-norms/norms on the energy space $\mathbf{E}$.

$$
\begin{aligned}
|\mathbf{v}|_{1, h} & :=\left(\sum_{K \in \mathcal{T}_{h}} \lambda\|\operatorname{div} \mathbf{v}\|_{L^{2}(K)}^{2}+2 \mu\|\varepsilon(\mathbf{v})\|_{L^{2}(K)}^{2}\right)^{\frac{1}{2}}, \\
\|\mathbf{v}\|_{1, h} & :=\left(|\mathbf{v}|_{1, h}^{2}+\sum_{e \in \mathcal{E}_{h}^{I}}\left(\frac{\gamma_{0, e}}{h_{e}}\|[\mathbf{v}]\|_{L^{2}(e)}^{2}+\gamma_{1, e} h_{e}\left\|\left[\sigma(\mathbf{v}) \mathbf{n}_{e}\right]\right\|_{L^{2}(e)}^{2}\right)\right)^{\frac{1}{2}}, \\
\|\mathbf{v}\|_{1, h} & :=\left(\|\mathbf{v}\|_{1, h}^{2}+\sum_{e \in \mathcal{E}_{h}^{I}} \frac{h_{e}}{\gamma_{0, e}}\left\|\left\{\sigma(\mathbf{v}) \mathbf{n}_{e}\right\}\right\|_{L^{2}(e)}^{2}\right)^{\frac{1}{2}} .
\end{aligned}
$$

The weak formulation for $(1.1)-(1.2)$ based on the sesquilinear form $a_{h}(\cdot, \cdot)$ is then defined as seeking $\mathbf{u} \in \mathbf{E} \cap \mathbf{H}_{\mathrm{loc}}^{2}(\Omega) \cap \mathbf{H}^{1}(\Omega)$ such that for all $\mathbf{v} \in \mathbf{E} \cap \mathbf{H}_{\text {loc }}^{2}(\Omega) \cap \mathbf{H}^{1}(\Omega)$

$$
a_{h}(\mathbf{u}, \mathbf{v})-\omega^{2} \rho(\mathbf{u}, \mathbf{v})_{\Omega}+\mathbf{i} \omega\langle A \mathbf{u}, \mathbf{v}\rangle_{\Gamma}=(\mathbf{f}, \mathbf{v})_{\Omega}+\langle\mathbf{g}, \mathbf{v}\rangle_{\Gamma} .
$$


Our discontinuous Galerkin finite element space is taken as the standard piecewise linear polynomial space

$$
\mathbf{V}_{h}:=\prod_{K \in \mathcal{T}_{h}} \mathbf{P}_{1}(K):=\prod_{K \in \mathcal{T}_{h}}\left(P_{1}(K)\right)^{d},
$$

where $P_{1}(K)$ is the space of linear polynomials with complex coefficients on the triangle/tetrahedron $K \in \mathcal{T}_{h}$. Then our IP-DG method is defined as seeking $\mathbf{u}_{h} \in \mathbf{V}_{h}$ such that

$$
A_{h}\left(\mathbf{u}_{h}, \mathbf{v}_{h}\right)=\left(\mathbf{f}, \mathbf{v}_{h}\right)_{\Omega}+\left\langle\mathbf{g}, \mathbf{v}_{h}\right\rangle_{\Gamma} \quad \forall \mathbf{v}_{h} \in \mathbf{V}_{h},
$$

where

$$
A_{h}\left(\mathbf{u}_{h}, \mathbf{v}_{h}\right):=a_{h}\left(\mathbf{u}_{h}, \mathbf{v}_{h}\right)-\omega^{2} \rho\left(\mathbf{u}_{h}, \mathbf{v}_{h}\right)_{\Omega}+\mathbf{i} \omega\left\langle A \mathbf{u}_{h}, \mathbf{v}_{h}\right\rangle_{\Gamma}
$$

In the following sections we shall establish stability and error estimates for the IP-DG method defined above. To prove these estimates we shall require that there exist $h, \gamma_{0}$, and $\gamma_{1}>0$ such that

$$
\begin{array}{cl}
h_{K} \leq h \leq C h_{K} & \forall K \in \mathcal{T}_{h}, \\
\gamma_{0, e} \leq \gamma_{0} \leq C \gamma_{0, e} & \forall e \in \mathcal{E}_{h}^{I}, \\
\gamma_{1, e} \leq \gamma_{1} \leq C \gamma_{1, e} & \forall e \in \mathcal{E}_{h}^{I},
\end{array}
$$

for some positive constant $C$ independent of $h$. It will also be convenient to introduce the notation $\xi:=1+\gamma_{0}^{-1}$. It is easy to show the following norm equivalence on $\mathbf{V}_{h}$ :

$$
\left\|\mathbf{v}_{h}\right\|_{1, h} \leq\left\|\mid \mathbf{v}_{h}\right\|_{1, h} \leq C \xi^{\frac{1}{2}}\left\|\mathbf{v}_{h}\right\|_{1, h} \quad \forall \mathbf{v}_{h} \in \mathbf{V}_{h} .
$$

4. Stability estimates. The goal of this section is to establish unconditional stability estimates for the solution of our IP-DG method, which can be regarded as the discrete counterpart of the following frequency-explicit estimate for the solution $\mathbf{u} \in \mathbf{H}^{2}(\Omega)$ of the problem $1.1-1.2$, which was established in [5].

THEOREM 4.1. Suppose that $\Omega$ is a convex polygonal domain or a smooth domain and $\mathbf{u} \in \mathbf{H}^{2}(\Omega)$ solves (1.1)- 1.2 . Then $\mathbf{u}$ satisfies the following estimate:

$$
\|\mathbf{u}\|_{H^{2}(\Omega)} \leq C\left(\omega^{\alpha}+\frac{1}{\omega^{2}}\right)\left(\|\mathbf{f}\|_{L^{2}(\Omega)}+\|\mathbf{g}\|_{L^{2}(\Gamma)}\right),
$$

where $\alpha=1$ if there exists $\tilde{K}>0$ such that $\mathbf{u} \in \mathbf{V}_{\tilde{K}}$ as defined in Section 2, and $\alpha=2$ otherwise.

REMARK 4.2. The desired Korn-type inequality on the boundary $\Gamma$ of a general domain $\Omega$ required for $\mathbf{u} \in \mathbf{V}_{\tilde{K}}$ is yet be proved and is posed as a conjecture in [5], although there are strong evidences to indicate that this is the case. Moreover, we believe that the optimal dependence on $\omega$ (i.e., when $\alpha=1$ ) should hold in (4.1) as that is is the case for the scalar Helmholtz equation and the time-Harmonic Maxwell's equations. For the rest of this paper we shall keep this parameter $\alpha$.

In [13], an a priori error estimate is obtained in the case $\omega^{1+\alpha} h=O(1)$ (called the asymptotic mesh regime), with stability being a consequence. This analysis is based on the so-called Schatz argument [15]. Similar results in the asymptotic mesh regime have been proved for the standard finite element method applied to the scalar and 
elastic Helmholtz equations (c.f. [4, 1, 6, 47). In this paper we are more interested in deriving solution estimates in the pre-asymptotic mesh regime, i.e. $\omega^{\beta} h=O(1)$ for some $1 \leq \beta<1+\alpha$. This is the most interesting case because similar results have not been proved for the standard finite element method for the elastic Helmholtz problem and the analysis requires a non-standard argument.

We begin by first proving some discrete weak coercivity properties for the sesquilinear form $A_{h}(\cdot, \cdot)$. The desired stability estimates can then be obtained as a consequence of these discrete weak coercivity properties.

Let us start with a technical lemma which will be instrumental in establishing the discrete coercivity of the sesquilinear form $A_{h}(\cdot, \cdot)$. We note that this lemma makes use of the fact that the space $\mathbf{V}_{h}$ is made up of piecewise linear polynomials, and thus a different strategy must be sought in the case that $\mathbf{V}_{h}$ is a higher order DG finite element space.

Lemma 4.3. For any $0<\delta<1$ and $\mathbf{v}_{h} \in \mathbf{V}_{h}$ there holds

$$
\begin{aligned}
\left|\mathbf{v}_{h}\right|_{1, h}^{2} \leq \delta & \omega^{2} \rho\left\|\mathbf{v}_{h}\right\|_{L^{2}(\Omega)}^{2}+\frac{C_{\delta}}{\omega h} \omega\left\|\mathbf{v}_{h}\right\|_{L^{2}(\Gamma)}^{2}+\frac{C_{\delta}}{\gamma_{0}} J_{0}\left(\mathbf{v}_{h}, \mathbf{v}_{h}\right) \\
& +\frac{C_{\delta}}{\omega^{2} \rho h^{2} \gamma_{1}} J_{1}\left(\mathbf{v}_{h}, \mathbf{v}_{h}\right)
\end{aligned}
$$

where $C$ is a positive constant independent of $\omega, h, \gamma_{0}$, and $\gamma_{1}$.

Proof. We note that $\left.\mathbf{v}_{h}\right|_{K} \in \mathbf{P}_{1}(K)$ for all $K \in \mathcal{T}_{h}$ and thus $\left.\operatorname{div} \sigma\left(\mathbf{v}_{h}\right)\right|_{K}=\mathbf{0}$. For any $\mathbf{w}_{h}, \mathbf{v}_{h} \in \mathbf{V}_{h}$ and $K \in \mathcal{T}_{h}$, integrating by parts yields

$$
\begin{aligned}
0 & =\left(\operatorname{div} \sigma\left(\mathbf{v}_{h}\right), \mathbf{w}_{h}\right)_{K} \\
& =-\left\langle\sigma\left(\mathbf{v}_{h}\right) \mathbf{n}_{K}, \mathbf{w}_{h}\right\rangle_{\partial K}+\lambda\left(\operatorname{div} \mathbf{v}_{h}, \operatorname{div} \mathbf{w}_{h}\right)_{K}+2 \mu\left(\varepsilon\left(\mathbf{v}_{h}\right), \varepsilon\left(\mathbf{w}_{h}\right)\right)_{K} .
\end{aligned}
$$

Here we have used a differential identity from Lemma 3 of [5], namely

$$
\sigma\left(\mathbf{v}_{h}\right): \nabla \overline{\mathbf{w}_{h}}=\lambda \operatorname{div} \mathbf{v}_{h} \operatorname{div} \overline{\mathbf{w}_{h}}+2 \mu \varepsilon\left(\mathbf{v}_{h}\right): \varepsilon\left(\overline{\mathbf{w}_{h}}\right) .
$$

Thus

$$
\lambda\left(\operatorname{div} \mathbf{v}_{h}, \operatorname{div} \mathbf{w}_{h}\right)_{K}+2 \mu\left(\varepsilon\left(\mathbf{v}_{h}\right), \varepsilon\left(\mathbf{w}_{h}\right)\right)_{K}=\left\langle\sigma\left(\mathbf{v}_{h}\right) \mathbf{n}_{K}, \mathbf{w}_{h}\right\rangle_{\partial K} .
$$

Setting $\mathbf{w}_{h}=\mathbf{v}_{h}$ and summing over all $K \in \mathcal{T}_{h}$ gives

$$
\begin{aligned}
\left|\mathbf{v}_{h}\right|_{1, h}^{2}= & \sum_{K \in \mathcal{T}_{h}}\left\langle\sigma\left(\mathbf{v}_{h}\right) \mathbf{n}_{K}, \mathbf{v}_{h}\right\rangle_{\partial K} \\
= & \sum_{e \in \mathcal{E}_{h}^{I}}\left(\left\langle\left\{\sigma\left(\mathbf{v}_{h}\right) \mathbf{n}_{e}\right\},\left[\mathbf{v}_{h}\right]\right\rangle_{e}+\left\langle\left[\sigma\left(\mathbf{v}_{h}\right) \mathbf{n}_{e}\right],\left\{\mathbf{v}_{h}\right\}\right\rangle_{e}\right)+\sum_{e \in \mathcal{E}_{h}^{B}}\left\langle\sigma\left(\mathbf{v}_{h}\right) \mathbf{n}_{e}, \mathbf{v}_{h}\right\rangle_{e} \\
\leq & \sum_{e \in \mathcal{E}_{h}^{I}}\left(\left\|\left\{\sigma\left(\mathbf{v}_{h}\right) \mathbf{n}_{e}\right\}\right\|_{L^{2}(e)}\left\|\left[\mathbf{v}_{h}\right]\right\|_{L^{2}(e)}+\left\|\left[\sigma\left(\mathbf{v}_{h}\right) \mathbf{n}_{e}\right]\right\|_{L^{2}(e)}\left\|\left\{\mathbf{v}_{h}\right\}\right\|_{L^{2}(e)}\right) \\
& \quad+\sum_{e \in \mathcal{E}_{h}^{B}}\left\|\sigma\left(\mathbf{v}_{h}\right) \mathbf{n}_{e}\right\|_{L^{2}(e)}\left\|\mathbf{v}_{h}\right\|_{L^{2}(e)} .
\end{aligned}
$$


Applying the trace and inverse inequalities on the right-hand side yields

$$
\begin{aligned}
\left|\mathbf{v}_{h}\right|_{1, h}^{2} \leq C & \sum_{e \in \mathcal{E}_{h}^{B}} h_{e}^{-\frac{1}{2}}\left\|\sigma\left(\mathbf{v}_{h}\right)\right\|_{L^{2}\left(K_{e}\right)}\left\|\mathbf{v}_{h}\right\|_{L^{2}(e)} \\
& +C \sum_{e \in \mathcal{E}_{h}^{I}} h_{e}^{-\frac{1}{2}}\left\|\left[\mathbf{v}_{h}\right]\right\|_{L^{2}(e)}\left(\left\|\sigma\left(\mathbf{v}_{h}\right)\right\|_{L^{2}\left(K_{e}\right)}+\left\|\sigma\left(\mathbf{v}_{h}\right)\right\|_{L^{2}\left(K_{e}^{\prime}\right)}\right) \\
& +C \sum_{e \in \mathcal{E}_{h}^{I}} h_{e}^{-\frac{1}{2}}\left\|\left[\sigma\left(\mathbf{v}_{h}\right) \mathbf{n}_{e}\right]\right\|_{L^{2}(e)}\left(\left\|\mathbf{v}_{h}\right\|_{L^{2}\left(K_{e}\right)}+\left\|\mathbf{v}_{h}\right\|_{L^{2}\left(K_{e}^{\prime}\right)}\right) .
\end{aligned}
$$

Finally, it follows from the discrete Cauchy-Schwarz inequality along with Young's inequality that

$$
\begin{aligned}
\left|\mathbf{v}_{h}\right|_{1, h}^{2} \leq & C h^{-\frac{1}{2}}\left(\sum_{K \in \mathcal{T}_{h}}\left\|\sigma\left(\mathbf{v}_{h}\right)\right\|_{L^{2}(K)}\right)^{\frac{1}{2}}\left\|\mathbf{v}_{h}\right\|_{L^{2}(\partial \Omega)} \\
& +C \gamma_{0}^{-\frac{1}{2}}\left(\sum_{e \in \mathcal{E}_{h}^{I}} \frac{\gamma_{0, e}}{h_{e}}\left\|\left[\mathbf{v}_{h}\right]\right\|_{L^{2}(e)}^{2}\right)^{\frac{1}{2}}\left(\sum_{K \in \mathcal{T}_{h}}\left\|\sigma\left(\mathbf{v}_{h}\right)\right\|_{L^{2}(K)}^{2}\right)^{\frac{1}{2}} \\
& +C \gamma_{1}^{-\frac{1}{2}} h^{-1}\left(\sum_{e \in \mathcal{E}_{h}^{I}} \gamma_{1, e} h_{e}\left\|\left[\sigma\left(\mathbf{v}_{h}\right) \mathbf{n}_{e}\right]\right\|_{L^{2}(e)}^{2}\right)^{\frac{1}{2}}\left\|\mathbf{v}_{h}\right\|_{L^{2}(\Omega)} \\
\leq \delta\left|\mathbf{v}_{h}\right|_{1, h}^{2} & +\frac{C(\lambda+2 \mu)}{\delta \omega h} \omega\left\|\mathbf{v}_{h}\right\|_{L^{2}(\partial \Omega)}^{2}+\frac{\delta}{2}\left|\mathbf{v}_{h}\right|_{1, h}^{2}+\frac{C(\lambda+2 \mu)}{\delta \gamma_{0}} J_{0}\left(\mathbf{v}_{h}, \mathbf{v}_{h}\right) \\
& +\delta(1-\delta) \omega^{2} \rho\left\|\mathbf{v}_{h}\right\|_{L^{2}(\Omega)}^{2}+\frac{C}{\delta(1-\delta) \omega^{2} \rho h^{2} \gamma_{1}} J_{1}\left(\mathbf{v}_{h}, \mathbf{v}_{h}\right) .
\end{aligned}
$$

Thus 4.2 holds.

We are now ready to state the following main theorem of this section.

TheOREM 4.4. Let $A_{h}(\cdot, \cdot)$ be the sesquilinear form defined in (3.7). Then there hold for all $\mathbf{v}_{h} \in \mathbf{V}_{h}$

$$
\begin{aligned}
& \left|A_{h}\left(\mathbf{v}_{h}, \mathbf{v}_{h}\right)\right| \geq C\left(\xi+\frac{1}{\omega h}+\frac{1}{\omega^{2} h^{2} \gamma_{1}}\right)^{-1}\left(\left|\mathbf{v}_{h}\right|_{1, h}^{2}+\omega^{2} \rho\left\|\mathbf{v}_{h}\right\|_{L^{2}(\Omega)}^{2}\right), \\
& \left|A_{h}\left(\mathbf{v}_{h}, \mathbf{v}_{h}\right)\right| \geq J_{0}\left(\mathbf{v}_{h}, \mathbf{v}_{h}\right)+J_{1}\left(\mathbf{v}_{h}, \mathbf{v}_{h}\right)+\omega\left\langle A \mathbf{v}_{h}, \mathbf{v}_{h}\right\rangle_{\Gamma},
\end{aligned}
$$

where $\xi=1+\gamma_{0}^{-1}$ and $C$ is a positive constant independent of $\omega, h, \gamma_{0}$, and $\gamma_{1}$.

Proof. Let $\mathbf{v}_{h} \in \mathbf{V}_{h}$. Taking the real and imaginary parts of $A_{h}\left(\mathbf{v}_{h}, \mathbf{v}_{h}\right)$ yields

$$
\begin{aligned}
& \operatorname{Re} A_{h}\left(\mathbf{v}_{h}, \mathbf{v}_{h}\right)=\left|\mathbf{v}_{h}\right|_{1, h}^{2}-\omega^{2} \rho\left\|\mathbf{v}_{h}\right\|_{L^{2}(\Omega)}^{2}-2 \operatorname{Re} \sum_{e \in \mathcal{E}_{h}^{I}}\left\langle\left\{\sigma\left(\mathbf{v}_{h}\right) \mathbf{n}_{e}\right\},\left[\mathbf{v}_{h}\right]\right\rangle_{e}, \\
& \operatorname{Im} A_{h}\left(\mathbf{v}_{h}, \mathbf{v}_{h}\right)=J_{0}\left(\mathbf{v}_{h}, \mathbf{v}_{h}\right)+J_{1}\left(\mathbf{v}_{h}, \mathbf{v}_{h}\right)+\omega\left\langle A \mathbf{v}_{h}, \mathbf{v}_{h}\right\rangle_{\Gamma} .
\end{aligned}
$$

Then (4.5) follows directly from (4.7).

To verify (4.4), we need to bound the term $\sum_{e \in \mathcal{E}_{h}^{I}}\left\langle\left\{\sigma\left(\mathbf{v}_{h}\right) \mathbf{n}_{e}\right\},\left[\mathbf{v}_{h}\right]\right\rangle_{e}$, which involves using the trace and inverse inequality and was already carried out previously in the proof of Lemma 4.3 . Thus

$$
\begin{aligned}
\operatorname{Re} A_{h}\left(\mathbf{v}_{h}, \mathbf{v}_{h}\right) & \leq\left|\mathbf{v}_{h}\right|_{1, h}^{2}-\omega^{2} \rho\left\|\mathbf{v}_{h}\right\|_{L^{2}(\Omega)}^{2}+C \gamma_{0}^{-\frac{1}{2}}\left(\sum_{e \in \mathcal{E}_{h}^{I}} \frac{\gamma_{0}}{h_{e}}\left\|\left[\mathbf{v}_{h}\right]\right\|_{L^{2}(\Omega)}^{2}\right)^{\frac{1}{2}}\left|\mathbf{v}_{h}\right|_{1, h} \\
& \leq \frac{3}{2}\left|\mathbf{v}_{h}\right|_{1, h}^{2}-\omega^{2} \rho\left\|\mathbf{v}_{h}\right\|_{L^{2}(\Omega)}^{2}+\frac{C}{\gamma_{0}} J_{0}\left(\mathbf{v}_{h}, \mathbf{v}_{h}\right)
\end{aligned}
$$


Combining the above inequality with 4.2 and using $\delta=\frac{1}{4}$ we get

$$
\begin{gathered}
\frac{1}{2}\left|\mathbf{v}_{h}\right|_{1, h}^{2}+\omega^{2} \rho\left\|\mathbf{v}_{h}\right\|_{L^{2}(\Omega)}^{2} \leq-\operatorname{Re} A_{h}\left(\mathbf{v}_{h}, \mathbf{v}_{h}\right)+2\left|\mathbf{v}_{h}\right|_{1, h}^{2}+\frac{C}{\gamma_{0}} J_{0}\left(\mathbf{v}_{h}, \mathbf{v}_{h}\right) \\
\leq- \\
\operatorname{Re} A_{h}\left(\mathbf{v}_{h}, \mathbf{v}_{h}\right)+\frac{1}{2} \omega^{2} \rho\left\|\mathbf{v}_{h}\right\|_{L^{2}(\Omega)}^{2}+\frac{C}{\omega h c_{A}} \omega c_{A}\left\|\mathbf{v}_{h}\right\|_{L^{2}(\partial \Omega)}^{2} \\
+\frac{C}{\gamma_{0}} J_{0}\left(\mathbf{v}_{h}, \mathbf{v}_{h}\right)+\frac{C}{\omega^{2} \rho h^{2} \gamma_{1}} J_{1}\left(\mathbf{v}_{h}, \mathbf{v}_{h}\right) .
\end{gathered}
$$

Thus, subtracting both sides of the above inequality by $\frac{1}{2} \omega^{2} \rho\left\|\mathbf{v}_{h}\right\|_{L^{2}(\Omega)}^{2}$ and using both 2.6 and 4.7) yield

$$
\begin{aligned}
\left|\mathbf{v}_{h}\right|_{1, h}^{2} & +\omega^{2} \rho\left\|\mathbf{v}_{h}\right\|_{L^{2}(\Omega)}^{2} \\
& \leq-2 \operatorname{Re} A_{h}\left(\mathbf{v}_{h}, \mathbf{v}_{h}\right)+C\left(\frac{1}{\gamma_{0}}+\frac{1}{\omega h c_{A}}+\frac{1}{\omega^{2} \rho h^{2} \gamma_{1}}\right) \operatorname{Im} A_{h}\left(\mathbf{v}_{h}, \mathbf{v}_{h}\right) \\
& \leq C\left(1+\frac{1}{\gamma_{0}}+\frac{1}{\omega h c_{A}}+\frac{1}{\omega^{2} \rho h^{2} \gamma_{1}}\right)\left|A_{h}\left(\mathbf{v}_{h}, \mathbf{v}_{h}\right)\right| .
\end{aligned}
$$

Hence, (4.4) holds. The proof is complete.

REMARK 4.5. (4.4-4.5 is called weak coercivity because of the complex magnitude (instead of the real part) is used in the left-hand sides of these inequalities.

As a consequence of the discrete weak coercivity of Theorem 4.4 we readily obtain the following stability estimates.

TheOREM 4.6. Suppose that $\mathbf{u}_{h} \in \mathbf{V}_{h}$ solves the IP-DG method given by (3.6). Then the following inequalities hold:

$$
\begin{aligned}
& \left|\mathbf{u}_{h}\right|_{1, h}^{2}+\omega^{2} \rho\left\|\mathbf{u}_{h}\right\|_{L^{2}(\Omega)}^{2} \leq C\left(C_{\text {sta }}^{2}\|\mathbf{f}\|_{L^{2}(\Omega)}^{2}+C_{\text {sta }}\|\mathbf{g}\|_{L^{2}(\Gamma)}^{2}\right) \\
& J_{0}\left(\mathbf{u}_{h}, \mathbf{u}_{h}\right)+J_{1}\left(\mathbf{u}_{h}, \mathbf{u}_{h}\right)+\left\langle A \mathbf{u}_{h}, \mathbf{u}_{h}\right\rangle_{\Gamma} \leq \frac{C}{\omega}\left(C_{\text {sta }}\|\mathbf{f}\|_{L^{2}(\Omega)}^{2}+\|\mathbf{g}\|_{L^{2}(\Gamma)}^{2}\right),
\end{aligned}
$$

where

$$
C_{\text {sta }}=\frac{\xi}{\omega}+\frac{1}{\omega^{2} h}+\frac{1}{\omega^{3} h^{2} \gamma_{1}}, \quad \xi=1+\frac{1}{\gamma_{0}},
$$

and $C$ is a positive constant independent of $\omega, h, \gamma_{0}$, and $\gamma_{1}$.

Proof. By 4.4 and 4.5 we get

$$
\begin{gathered}
\left|\mathbf{u}_{h}\right|_{1, h}^{2}+\omega^{2} \rho\left\|\mathbf{u}_{h}\right\|_{L^{2}(\Omega)}^{2}+\omega\left(1+\frac{1}{\gamma_{0}}+\frac{1}{\omega h}+\frac{1}{\omega^{2} \rho h^{2} \gamma_{1}}\right)\left\langle A \mathbf{u}_{h}, \mathbf{u}_{h}\right\rangle_{\Gamma} \\
\leq C\left(1+\frac{1}{\gamma_{0}}+\frac{1}{\omega h}+\frac{1}{\omega^{2} \rho h^{2} \gamma_{1}}\right)\left|A_{h}\left(\mathbf{u}_{h}, \mathbf{u}_{h}\right)\right| \\
\leq C\left(1+\frac{1}{\gamma_{0}}+\frac{1}{\omega h}+\frac{1}{\omega^{2} \rho h^{2} \gamma_{1}}\right)\left(\|\mathbf{f}\|_{L^{2}(\Omega)}\left\|\mathbf{u}_{h}\right\|_{L^{2}(\Omega)}+\|\mathbf{g}\|_{L^{2}(\Gamma)}\left\|\mathbf{u}_{h}\right\|_{L^{2}(\Gamma)}\right) \\
\leq \frac{C}{\omega^{2} \rho}\left(1+\frac{1}{\gamma_{0}}+\frac{1}{\omega h}+\frac{1}{\omega^{2} \rho h^{2} \gamma_{1}}\right)^{2}\|\mathbf{f}\|_{L^{2}(\Omega)}^{2}+\frac{\omega^{2} \rho}{2}\left\|\mathbf{u}_{h}\right\|_{L^{2}(\Omega)}^{2} \\
\quad+\frac{C}{\omega c_{A}}\left(1+\frac{1}{\gamma_{0}}+\frac{1}{\omega h}+\frac{1}{\omega^{2} \rho h^{2} \gamma_{1}}\right)\|\mathbf{g}\|_{L^{2}(\Gamma)}^{2} \\
+\omega c_{A}\left(1+\frac{1}{\gamma_{0}}+\frac{1}{\omega h}+\frac{1}{\omega^{2} \rho h^{2} \gamma_{1}}\right)\left\|\mathbf{u}_{h}\right\|_{L^{2}(\Gamma)}^{2}
\end{gathered}
$$


Substituting (2.6) into the above inequality infers 4.8.

Now, combining (4.5) with 4.8 yields

$$
\begin{aligned}
J_{0}\left(\mathbf{u}_{h},\right. & \left.\mathbf{u}_{h}\right)+J_{1}\left(\mathbf{u}_{h}, \mathbf{u}_{h}\right)+\omega\left\langle A \mathbf{u}_{h}, \mathbf{u}_{h}\right\rangle_{\Gamma} \\
& \leq\left|A_{h}\left(\mathbf{u}_{h}, \mathbf{u}_{h}\right)\right| \\
& \leq\|\mathbf{f}\|_{L^{2}(\Omega)}\left\|\mathbf{u}_{h}\right\|_{L^{2}(\Omega)}+\|\mathbf{g}\|_{L^{2}(\Gamma)}\left\|\mathbf{u}_{h}\right\|_{L^{2}(\Gamma)} \\
\leq & \frac{C}{\omega \rho^{\frac{1}{2}}}\|\mathbf{f}\|_{L^{2}(\Omega)}\left(\frac{1}{\rho} C_{\text {sta }}^{2}\|\mathbf{f}\|_{L^{2}(\Omega)}^{2}+\frac{1}{c_{A}} C_{\text {sta }}\|\mathbf{g}\|_{L^{2}(\Gamma)}^{2}\right)^{\frac{1}{2}} \\
& \quad+\frac{C}{\omega c_{A}}\|\mathbf{g}\|_{L^{2}(\Gamma)}^{2}+\frac{\omega c_{A}}{2}\left\|\mathbf{u}_{h}\right\|_{L^{2}(\Gamma)}^{2} \\
\leq & \frac{C}{\omega \rho} C_{\text {sta }}\|\mathbf{f}\|_{L^{2}(\Omega)}^{2}+\frac{C}{\omega c_{A}}\|\mathbf{g}\|_{L^{2}(\Gamma)}^{2}+\frac{\omega c_{A}}{2}\left\|\mathbf{u}_{h}\right\|_{L^{2}(\Gamma)}^{2} .
\end{aligned}
$$

Using (2.6) in the above inequality infers the desired estimate 4.9 ).

REMARK 4.7. When $h$ is restricted to the pre-asymptotic mesh regime characterized by the constraint $\omega^{\beta} h=O(1)$ for $1 \leq \beta<1+\alpha$, then we have

$$
C_{\text {sta }} \leq C\left(\frac{\xi}{\omega}+\omega^{\beta-2}+\frac{\omega^{2 \beta-3}}{\gamma_{1}}\right) .
$$

Thus, the constant in the above stability estimate can be replaced by one that is independent of $h$ (but still depends on $\Omega$ ) in the pre-asymptotic mesh regime.

We conclude this section by establishing the following existence and uniqueness theorem.

Theorem 4.8. For any $\omega, h, \gamma_{0}, \gamma_{1}>0, \mathbf{f} \in \mathbf{L}^{2}(\Omega)$, and $\mathbf{g} \in \mathbf{L}^{2}(\partial \Omega)$ there exists a unique solution $\mathbf{u}_{h}$ of (3.6).

Proof. Due to the linearity of the PDE, it is easy to check that (3.6) is equivalent to a linear (algebraic) system. The stability estimate 4.8 immediately implies that the linear system only has the trivial solution when $\mathbf{f}=\mathbf{0}$ and $\mathbf{g}=\mathbf{0}$, which then infers that the coefficient matrix of the linear system is nonsingular. Thus, the system must have a unique solution and consequently (3.6) has a unique solution.

5. Error estimates. The goal of this section is to provide error estimates that hold in all mesh regimes, in particular, in the pre-asymptotic mesh regime. In order to accomplish this we shall adapt a non-standard argument of [9, 11] by defining an elliptic projection operator onto $\mathbf{V}_{h}$ and establishing estimates for the error between the solution $\mathbf{u} \in \mathbf{E} \cap \mathbf{H}^{d}(\Omega)$ of $(1.1)-(1.2)$ and its elliptic projection, and utilizing the unconditional stability estimate to control the error between the projection and the IP-DG solution. In this section we assume $\mathbf{u} \in \mathbf{H}^{2}(\Omega)$ satisfying the estimate given in Theorem 4.1 .

First, we show that the sesquilinear form $a_{h}(\cdot, \cdot)$ is continuous and satisfies a weak coercivity condition.

TheOREM 5.1. For any $\mathbf{v}, \mathbf{w} \in \mathbf{E}$ there exists a positive constant $C$ independent of $\omega, h, \gamma_{0}, \gamma_{1}$ such that

$$
\left|a_{h}(\mathbf{v}, \mathbf{w})\right| \leq\left. C|||\mathbf{v}|||_{1, h}|||\mathbf{w}|\right|_{1, h} .
$$

Also for any $0<\delta<1$ and $\mathbf{v}_{h} \in \mathbf{V}_{h}$ there holds

$$
\operatorname{Re} a_{h}\left(\mathbf{v}_{h}, \mathbf{v}_{h}\right)+\left(1-\delta+\frac{C_{\delta}}{\gamma_{0}}\right) \operatorname{Im} a_{h}\left(\mathbf{v}_{h}, \mathbf{v}_{h}\right) \geq(1-\delta)\left\|\mathbf{v}_{h}\right\|_{1, h}^{2} .
$$


Proof. (5.1) is easy to prove, we omit it and leave it to the interested reader. To show (5.2), we note that

$$
\begin{aligned}
\operatorname{Re} a_{h}\left(\mathbf{v}_{h}, \mathbf{v}_{h}\right) & \geq\left|\mathbf{v}_{h}\right|_{1, h}^{2}+2 \sum_{e \in \mathcal{E}_{h}^{I}}\left|\left\langle\left[\mathbf{v}_{h}\right],\left\{\sigma\left(\mathbf{v}_{h}\right) \mathbf{n}_{e}\right\}\right\rangle_{e}\right| \\
& \geq\left|\mathbf{v}_{h}\right|_{1, h}^{2}-2 \sum_{e \in \mathcal{E}_{h}^{I}}\left\|\left[\mathbf{v}_{h}\right]\right\|_{L^{2}(e)}\left\|\left\{\sigma\left(\mathbf{v}_{h}\right) \mathbf{n}_{e}\right\}\right\|_{L^{2}(e)} \\
& \geq\left|\mathbf{v}_{h}\right|_{1, h}^{2}-\sum_{e \in \mathcal{E}_{h}^{I}} \frac{C}{\gamma_{0}} \frac{\gamma_{0}}{h_{e}^{\frac{1}{2}}}\left\|\left[\mathbf{v}_{h}\right]\right\|_{L^{2}(e)}\left(\left\|\sigma\left(\mathbf{v}_{h}\right)\right\|_{L^{2}\left(K_{e}\right)}+\left\|\sigma\left(\mathbf{v}_{h}\right)\right\|_{L^{2}\left(K_{e}^{\prime}\right)}\right) \\
& \geq(1-\delta)\left|\mathbf{v}_{h}\right|_{1, h}^{2}-\frac{C_{\delta}}{\gamma_{0}} J_{0}\left(\mathbf{v}_{h}, \mathbf{v}_{h}\right) .
\end{aligned}
$$

Also,

$$
\operatorname{Im} a_{h}\left(\mathbf{v}_{h}, \mathbf{v}_{h}\right)=J_{0}\left(\mathbf{v}_{h}, \mathbf{v}_{h}\right)+J_{1}\left(\mathbf{v}_{h}, \mathbf{v}_{h}\right) .
$$

5.2 follows from combining the above two results.

5.1. Elliptic projection and its error estimates. For any $\mathbf{w} \in E$ we define its elliptic projection $\tilde{\mathbf{w}}_{h} \in \mathbf{V}_{h}$ as the solution to the following problem:

$$
a_{h}\left(\tilde{\mathbf{w}}_{h}, \mathbf{v}_{h}\right)+\mathbf{i} \omega\left\langle A \tilde{\mathbf{w}}_{h}, \mathbf{v}_{h}\right\rangle_{\Gamma}=a_{h}\left(\mathbf{w}, \mathbf{v}_{h}\right)+\mathbf{i} \omega\left\langle A \mathbf{w}, \mathbf{v}_{h}\right\rangle_{\Gamma} \quad \forall \mathbf{v}_{h} \in \mathbf{V}_{h} .
$$

REMARK 5.2. By Theorem 5.1, the sesquilinear form $a_{h}(\cdot, \cdot)+\mathbf{i} \omega\langle A \cdot, \cdot\rangle_{\Gamma}$ is both continuous and coercive when $\gamma_{0}$ is taken to be sufficiently large. Thus, the LaxMilgram Theorem ensures that $\tilde{\mathbf{w}}_{h}$ is well-defined for such a choice of $\gamma_{0}$.

By the consistency of $a_{h}(\cdot, \cdot)$, it is trivial to check that the following Galerkin orthogonality property holds.

Lemma 5.3. Suppose that $\mathbf{w} \in \mathbf{E}$ and $\tilde{\mathbf{w}}_{h} \in \mathbf{V}_{h}$ is its elliptic projection, then

$$
a_{h}\left(\mathbf{w}-\tilde{\mathbf{w}}_{h}, \mathbf{v}_{h}\right)+\mathbf{i} \omega\left\langle A\left(\mathbf{w}-\tilde{\mathbf{w}}_{h}\right), \mathbf{v}_{h}\right\rangle=0 \quad \forall \mathbf{v}_{h} \in \mathbf{V}_{h} .
$$

Now we are ready to estimate the error between the solution $\mathbf{u}$ of $1.1--1.2)$ and its elliptic projection $\tilde{\mathbf{u}}_{h}$.

Theorem 5.4. Let $\mathbf{u} \in \mathbf{H}^{2}(\Omega)$ solve (1.1)-1.2) and let $\tilde{\mathbf{u}}_{h} \in \mathbf{V}_{h}$ be its elliptic projection defined in (5.3). Then there hold the following estimates:

$$
\begin{aligned}
& \|\| \mathbf{u}-\tilde{\mathbf{u}}_{h} \mid\left\|_{1, h}+\omega^{\frac{1}{2}} \xi\right\| \mathbf{u}-\tilde{\mathbf{u}}_{h} \|_{L^{2}(\Gamma)} \\
& \quad \leq C \xi^{2} h\left(\xi+\gamma_{1}+\omega h\right)^{\frac{1}{2}}\left(\omega^{\alpha}+\frac{1}{\omega^{2}}\right)\left(\|\mathbf{f}\|_{L^{2}(\Omega)}+\|\mathbf{g}\|_{L^{2}(\Gamma)}\right) . \\
& \left\|\mathbf{u}-\tilde{\mathbf{u}}_{h}\right\|_{L^{2}(\Omega)} \leq C \xi^{2} h^{2}\left(\xi+\gamma_{1}+\omega h\right)\left(\omega^{\alpha}+\frac{1}{\omega^{2}}\right)\left(\|\mathbf{f}\|_{L^{2}(\Omega)}+\|\mathbf{g}\|_{L^{2}(\Gamma)}\right),
\end{aligned}
$$

where $\xi=1+\gamma_{0}^{-1}$ and $C$ is a positive constant independent of $\omega, h, \gamma_{0}$, and $\gamma_{1}$.

Proof. Let $\hat{\mathbf{u}}_{h} \in \mathbf{V}_{\mathbf{h}}$ denote the $P_{1}$-conforming finite element interpolant of $\mathbf{u}$ on $\mathcal{T}_{h}$. Then the following estimates are well-known (c.f. [2, 3]):

$$
\begin{aligned}
\left\|\mathbf{u}-\hat{\mathbf{u}}_{h}\right\|_{L^{2}(\Omega)} & \leq C h^{2}|\mathbf{u}|_{H^{2}(\Omega)}, \\
\left\|\boldsymbol{\nabla}\left(\mathbf{u}-\hat{\mathbf{u}}_{h}\right)\right\|_{L^{2}(\Omega)} & \leq C h|\mathbf{u}|_{H^{2}(\Omega)} .
\end{aligned}
$$


By the trace and inverse inequalities we get

$$
\begin{aligned}
& \left\|\left|\mathbf{u}-\hat{\mathbf{u}}_{h}\right|\right\|_{1, h} \leq C\left(\xi+\gamma_{1}\right)^{\frac{1}{2}} h|\mathbf{u}|_{H^{2}(\Omega)}, \\
& \left\|\mathbf{u}-\hat{\mathbf{u}}_{h}\right\|_{L^{2}(\Gamma)} \leq C h^{\frac{3}{2}}|\mathbf{u}|_{H^{2}(\Omega)} .
\end{aligned}
$$

Set $\psi_{h}:=\tilde{\mathbf{u}}_{h}-\hat{\mathbf{u}}_{h}$. By Galerkin orthogonality along with the fact that $\boldsymbol{\psi}_{h}+\mathbf{u}-\tilde{\mathbf{u}}_{h}=$ $\mathbf{u}-\hat{\mathbf{u}}_{h}$ we obtain

$$
a_{h}\left(\boldsymbol{\psi}_{h}, \boldsymbol{\psi}_{h}\right)+\mathbf{i} \omega\left\langle A \boldsymbol{\psi}_{h}, \boldsymbol{\psi}_{h}\right\rangle_{\Gamma}=a_{h}\left(\mathbf{u}-\hat{\mathbf{u}}_{h}, \boldsymbol{\psi}_{h}\right)+\mathbf{i} \omega\left\langle A\left(\mathbf{u}-\hat{\mathbf{u}}_{h}\right), \boldsymbol{\psi}_{h}\right\rangle_{\Gamma} .
$$

Next, it follows from 3.8 and 5.2 with $\delta=\frac{1}{2}$ (noting that $C_{\frac{1}{2}}>\frac{1}{2}$ ) that

$$
\begin{aligned}
& \frac{1}{2} \mid\left\|\boldsymbol{\psi}_{h}\right\|_{1, h}^{2} \leq C \xi\left\|\boldsymbol{\psi}_{h}\right\|_{1, h}^{2} \\
& \leq C \xi \operatorname{Re} a_{h}\left(\boldsymbol{\psi}_{h}, \boldsymbol{\psi}_{h}\right)+C \xi\left(\frac{1}{2}+\frac{C_{\frac{1}{2}}}{\gamma_{0}}\right) \operatorname{Im} a_{h}\left(\boldsymbol{\psi}_{h}, \boldsymbol{\psi}_{h}\right) \\
& =C \xi \operatorname{Re}\left(a_{h}\left(\boldsymbol{\psi}_{h}, \boldsymbol{\psi}_{h}\right)+\mathbf{i} \omega\left\langle A \boldsymbol{\psi}_{h}, \boldsymbol{\psi}_{h}\right\rangle_{\Gamma}\right) \\
& +C \xi\left(\frac{1}{2}+\frac{C_{\frac{1}{2}}}{\gamma_{0}}\right) \operatorname{Im}\left(a_{h}\left(\boldsymbol{\psi}_{h}, \boldsymbol{\psi}_{h}\right)+\mathbf{i} \omega\left\langle A \boldsymbol{\psi}_{h}, \boldsymbol{\psi}_{h}\right\rangle_{\Gamma}\right) \\
& -C \xi \omega\left(\frac{1}{2}+\frac{C_{\frac{1}{2}}}{\gamma_{0}}\right)\left\langle A \boldsymbol{\psi}_{h}, \boldsymbol{\psi}_{h}\right\rangle_{\Gamma} \\
& =C \xi \operatorname{Re}\left(a_{h}\left(\mathbf{u}-\hat{\mathbf{u}}_{h}, \boldsymbol{\psi}_{h}\right)+\mathbf{i} \omega\left\langle A\left(\mathbf{u}-\hat{\mathbf{u}}_{h}\right), \boldsymbol{\psi}_{h}\right\rangle_{\Gamma}\right) \\
& +C \xi\left(\frac{1}{2}+\frac{C_{\frac{1}{2}}}{\gamma_{0}}\right) \operatorname{Im}\left(a_{h}\left(\mathbf{u}-\hat{\mathbf{u}}_{h}, \boldsymbol{\psi}_{h}\right)+\mathbf{i} \omega\left\langle A\left(\mathbf{u}-\hat{\mathbf{u}}_{h}\right), \boldsymbol{\psi}_{h}\right\rangle_{\Gamma}\right) \\
& -C \xi \omega\left(\frac{1}{2}+\frac{C_{\frac{1}{2}}}{\gamma_{0}}\right)\left\langle A \boldsymbol{\psi}_{h}, \boldsymbol{\psi}_{h}\right\rangle_{\Gamma} \\
& \leq C \xi\left|\left\|\boldsymbol { \psi } _ { h } \left|\left\|_{1, h} \mid\right\| \mathbf{u}-\hat{\mathbf{u}}\|\|_{1, h}+C \omega \xi\left\|\boldsymbol{\psi}_{h}\right\|_{L^{2}(\Gamma)}\|\mathbf{u}-\hat{\mathbf{u}}\|_{L^{2}(\Gamma)}-C \omega \xi^{2}\left\|\boldsymbol{\psi}_{h}\right\|_{L^{2}(\Gamma)}^{2}\right.\right.\right. \\
& +C \xi^{2}\left(\left\|\boldsymbol{\psi}_{h}\left|\left\|_{1, h} \mid\right\| \mathbf{u}-\hat{\mathbf{u}}\|\|_{1, h}+\omega\left\|\boldsymbol{\psi}_{h}\right\|_{L^{2}(\Gamma)}\|\mathbf{u}-\hat{\mathbf{u}}\|_{L^{2}(\Gamma)}\right)\right.\right. \\
& \leq C \xi^{2}\left\|\left|\boldsymbol{\psi}_{h}\left\|\left.\right|_{1, h} \mid\right\| \mathbf{u}-\hat{\mathbf{u}}\left\|_{1, h}+2 C \omega \xi^{2}\right\| \mathbf{u}-\hat{\mathbf{u}}_{h}\left\|_{L^{2}(\Gamma)}^{2}-\frac{C}{4} \omega \xi^{2}\right\| \boldsymbol{\psi}_{h} \|_{L^{2}(\Gamma)}\right.\right. \\
& \leq C \xi^{4}\|\| \mathbf{u}-\hat{\mathbf{u}}_{h}\left\|\left.\right|_{1, h} ^{2}+\frac{1}{4}\right\|\left|\boldsymbol{\psi}_{h}\left\|\left.\right|_{1, h} ^{2}-\frac{C}{4} \omega \xi^{2}\right\| \boldsymbol{\psi}_{h}\left\|_{L^{2}(\Gamma)}^{2}+2 C \omega \xi^{2}\right\| \mathbf{u}-\hat{\mathbf{u}}_{h} \|_{L^{2}(\Gamma)}^{2} .\right.
\end{aligned}
$$

Substituting (5.9) and (5.10) into the above estimate gives

$$
\begin{aligned}
\left\|\mid \boldsymbol{\psi}_{h}\right\| \|_{1, h}^{2} & +\omega \xi^{2}\left\|\boldsymbol{\psi}_{h}\right\|_{L^{2}(\Gamma)}^{2} \leq C\left(\xi^{4}\left|\left\|\mathbf{u}-\hat{\mathbf{u}}_{h} \mid\right\|_{1, h}^{2}+\omega \xi^{2}\left\|\mathbf{u}-\hat{\mathbf{u}}_{h}\right\|_{L^{2}(\Gamma)}^{2}\right)\right. \\
& \leq C\left(\xi^{4}\left(\xi+\gamma_{1}\right) h^{2}|\mathbf{u}|_{H^{2}(\Omega)}^{2}+\omega \xi^{2} h^{3}|\mathbf{u}|_{H^{2}(\Omega)}^{2}\right) \\
& =C \xi^{4} h^{2}\left(\xi+\gamma_{1}+\omega h\right)|\mathbf{u}|_{H^{2}(\Omega)}^{2} \\
& \leq C \xi^{4} h^{2}\left(\xi+\gamma_{1}+\omega h\right)\left(\omega^{\alpha}+\frac{1}{\omega^{2}}\right)^{2}\left(\|\mathbf{f}\|_{L^{2}(\Omega)}^{2}+\|\mathbf{g}\|_{L^{2}(\Gamma)}^{2}\right) .
\end{aligned}
$$

Thus

$$
\begin{aligned}
\|\| \boldsymbol{\psi}_{h} \mid \|_{1, h} & +\omega^{\frac{1}{2}} \xi\left\|\boldsymbol{\psi}_{h}\right\|_{L^{2}(\Gamma)} \\
& \leq C \xi^{2} h\left(\xi+\gamma_{1}+\omega h\right)^{\frac{1}{2}}\left(\omega^{\alpha}+\frac{1}{\omega^{2}}\right)\left(\|\mathbf{f}\|_{L^{2}(\Omega)}+\|\mathbf{g}\|_{L^{2}(\Gamma)}^{2}\right) .
\end{aligned}
$$


Recall that $\mathbf{u}-\tilde{\mathbf{u}}_{h}=\mathbf{u}-\hat{\mathbf{u}}_{h}-\boldsymbol{\psi}_{h}$. By the triangle inequality we get

$$
\begin{aligned}
\left.\left\|\left|\mathbf{u}-\tilde{\mathbf{u}}_{h}\right|\right\|\right|_{1, h}+\omega^{\frac{1}{2}} \xi\left\|\mathbf{u}-\tilde{\mathbf{u}}_{h}\right\|_{L^{2}(\Gamma)} & \\
& \leq\left\|\left|\mathbf{u}-\hat{\mathbf{u}}_{h}\right|\right\|_{1, h}+\omega^{\frac{1}{2}} \xi\left\|\mathbf{u}-\hat{\mathbf{u}}_{h}\right\|_{L^{2}(\Gamma)}+\|\| \boldsymbol{\psi}_{h}\|\|_{1, h}+\omega^{\frac{1}{2}} \xi\left\|\boldsymbol{\psi}_{h}\right\|_{L^{2}(\Gamma)} \\
& \leq C \xi^{2} h\left(\xi+\gamma_{1}+\omega h\right)^{\frac{1}{2}}\left(\omega^{\alpha}+\frac{1}{\omega^{2}}\right)\left(\|\mathbf{f}\|_{L^{2}(\Omega)}+\|\mathbf{g}\|_{L^{2}(\Gamma)}^{2}\right) .
\end{aligned}
$$

Therefore, (5.5) holds.

To obtain (5.6), we appeal to the duality argument by considering the following auxiliary PDE problem:

$$
\begin{aligned}
-\operatorname{div}(\sigma(\mathbf{w}))=\mathbf{u}-\tilde{\mathbf{u}}_{h} & \text { in } \Omega, \\
\sigma(\mathbf{w}) \mathbf{n}-\mathbf{i} \omega \mathbf{w}=\mathbf{0} & \text { on } \Gamma .
\end{aligned}
$$

It can be shown that there exists a unique solution $\mathbf{w} \in \mathbf{H}^{2}(\Omega)$ such that

$$
\|\mathbf{w}\|_{H^{2}(\Omega)} \leq C\left\|\mathbf{u}-\tilde{\mathbf{u}}_{h}\right\|_{L^{2}(\Omega)}
$$

Let $\hat{\mathbf{w}}_{h} \in \mathbf{V}_{h}$ denote the $P_{1}$-conforming finite element interpolant of $\mathbf{w}$ over $\mathcal{T}_{h}$. Then by testing the above auxiliary problem with $\mathbf{u}-\tilde{\mathbf{u}}_{h}$ we get

$$
\begin{aligned}
\| \mathbf{u}- & \tilde{\mathbf{u}}_{h} \|_{L^{2}(\Omega)}^{2}=-\left(\mathbf{u}-\tilde{\mathbf{u}}_{h}, \operatorname{div} \sigma(\mathbf{w})\right)_{\Omega} \\
& =a_{h}\left(\mathbf{u}-\tilde{\mathbf{u}}_{h}, \mathbf{w}\right)+\mathbf{i} \omega\left\langle A\left(\mathbf{u}-\tilde{\mathbf{u}}_{h}\right), \mathbf{w}\right\rangle_{\Gamma} \\
& =a_{h}\left(\mathbf{u}-\tilde{\mathbf{u}}_{h}, \mathbf{w}-\hat{\mathbf{w}}_{h}\right) \mathbf{i} \omega\left\langle A\left(\mathbf{u}-\tilde{\mathbf{u}}_{h}\right), \mathbf{w}-\hat{\mathbf{w}}_{h}\right\rangle_{\Gamma} \\
& \leq C\left(\left\|\mathbf{u}-\tilde{\mathbf{u}}_{h}\left|\|\|_{1, h}\left\|\mid \mathbf{w}-\hat{\mathbf{w}}_{h}\right\|\left\|_{1, h}+\omega\right\| \mathbf{u}-\tilde{\mathbf{u}}_{h}\left\|_{L^{2}(\Gamma)}\right\| \mathbf{w}-\hat{\mathbf{w}}_{h} \|_{L^{2}(\Omega)}\right)\right.\right. \\
& \leq C\left(\left(\xi+\gamma_{1}\right)^{\frac{1}{2}} h\|\mathbf{w}\|_{H^{2}(\Omega)}\left\|\left|\mathbf{u}-\tilde{\mathbf{u}}_{h}\right|\right\|_{1, h}+\omega h^{\frac{3}{2}}\|\mathbf{w}\|_{H^{2}(\Omega)}\left\|\mathbf{u}-\tilde{\mathbf{u}}_{h}\right\|_{L^{2}(\Gamma)}\right) \\
& \leq C\left(( \xi + \gamma _ { 1 } ) ^ { \frac { 1 } { 2 } } h \| \mathbf { u } - \tilde { \mathbf { u } } _ { h } \| _ { L ^ { 2 } ( \Omega ) } \left\|\left|\mathbf{u}-\tilde{\mathbf{u}}_{h}\left\|\left.\right|_{1, h}+\omega h^{\frac{3}{2}}\right\| \mathbf{u}-\tilde{\mathbf{u}}_{h}\left\|_{L^{2}(\Omega)}\right\| \mathbf{u}-\tilde{\mathbf{u}}_{h} \|_{L^{2}(\Gamma)}\right) .\right.\right.
\end{aligned}
$$

Hence,

$$
\begin{aligned}
\left\|\mathbf{u}-\tilde{\mathbf{u}}_{h}\right\|_{L^{2}(\Omega)} & \leq C h\left(\xi+\gamma_{1}+\omega h\right)^{\frac{1}{2}}\left(\left\|\mathbf{u}-\tilde{\mathbf{u}}_{h}\right\|_{1, h}+\omega^{\frac{1}{2}} \xi\left\|\mathbf{u}-\tilde{\mathbf{u}}_{h}\right\|_{L^{2}(\Omega)}\right) \\
& \leq C \xi^{2} h^{2}\left(\xi+\gamma_{1}+\omega h\right)\left(\omega^{\alpha}+\frac{1}{\omega^{2}}\right)\left(\|\mathbf{f}\|_{L^{2}(\omega)}+\|\mathbf{g}\|_{L^{2}(\Gamma)}^{2}\right) .
\end{aligned}
$$

Thus, 5.6 holds.

5.2. Error estimates for the IP-DG method. The goal of this subsection is to obtain upper bounds for the error $\mathbf{e}_{h}:=\mathbf{u}-\mathbf{u}_{h}$, where $\mathbf{u} \in \mathbf{H}^{2}(\Omega)$ solve 1.1 -1.2 and $\mathbf{u}_{h}$ is its IP-DG approximation defined by (3.6). We shall accomplish this goal by using the stability estimate from Theorem 4.6 and the elliptic projection studied in Subsection 5.1. Subtracting (3.6) from (3.5) immediately infers the following Galerkin orthogonality for $\mathbf{e}_{h}$ :

$$
a_{h}\left(\mathbf{e}_{h}, \mathbf{v}_{h}\right)-\omega^{2} \rho\left(\mathbf{e}_{h}, \mathbf{v}_{h}\right)_{\Omega}+\mathbf{i} \omega\left\langle A \mathbf{e}_{h}, \mathbf{v}_{h}\right\rangle_{\Gamma}=\mathbf{0} \quad \forall \mathbf{v}_{h} \in \mathbf{V}_{h} .
$$

Let $\tilde{\mathbf{u}}_{h} \in \mathbf{V}_{h}$ be the elliptic projection of $\mathbf{u}$ as defined in (5.3). Next we define $\boldsymbol{\psi}:=\mathbf{u}-\tilde{\mathbf{u}}_{h}$ and $\phi_{h}:=\tilde{\mathbf{u}}_{h}-\mathbf{u}_{h}$. Thus, $\mathbf{e}_{h}$ can be decomposed as $\mathbf{e}_{h}=\boldsymbol{\psi}+\boldsymbol{\phi}_{h}$. By 
Galerkin orthogonality given in (5.4) and 5.12, we have the following identity:

$$
\begin{aligned}
a_{h}\left(\phi_{h}, \mathbf{v}_{h}\right) & -\omega^{2} \rho\left(\phi_{h}, \mathbf{v}_{h}\right)_{\Omega}+\mathbf{i} \omega\left\langle A \phi_{h}, \mathbf{v}_{h}\right\rangle_{\Gamma} \\
& =-a_{h}\left(\boldsymbol{\psi}, \mathbf{v}_{h}\right)+\omega^{2} \rho\left(\boldsymbol{\psi}, \mathbf{v}_{h}\right)_{\Omega}-\mathbf{i} \omega\left\langle A \boldsymbol{\psi}, \mathbf{v}_{h}\right\rangle_{\Gamma} \\
& =\omega^{2} \rho\left(\boldsymbol{\psi}, \mathbf{v}_{h}\right)_{\Omega} \quad \forall \mathbf{v}_{h} \in \mathbf{V}_{h} .
\end{aligned}
$$

In other words we obtain that $\boldsymbol{\phi}_{h} \in \mathbf{V}_{h}$ solves (3.6) with $\mathbf{f}=\omega^{2} \rho \boldsymbol{\psi}$ and $\mathbf{g} \equiv \mathbf{0}$. This allows us to bound $\phi_{h}$ by using the estimates from Theorem 4.6 and Theorem 5.4 Specifically, we have the next lemma.

Lemma 5.5. Let $\mathbf{u} \in \mathbf{H}^{2}(\Omega)$ solve (1.1)-(1.2), $\mathbf{u}_{h}$ be its IP-DG approximation, and $\tilde{\mathbf{u}}_{h}$ its elliptic projection. Then $\phi_{h}:=\tilde{\mathbf{u}}_{h}-\mathbf{u}_{h}$ satisfies

$$
\begin{aligned}
\left\|\phi_{h}\right\|_{1, h}+ & \omega \rho^{\frac{1}{2}}\left\|\phi_{h}\right\|_{L^{2}(\Omega)} \\
& \leq C \xi^{2} \omega^{2} h^{2} C_{\text {sta }}\left(\xi+\gamma_{1}+\omega h\right)\left(\omega^{\alpha}+\frac{1}{\omega^{2}}\right)\left(\|\mathbf{f}\|_{L^{2}(\Omega)}+\|\mathbf{g}\|_{L^{2}(\Gamma)}\right) .
\end{aligned}
$$

Proof. As mentioned above, (5.13) implies that $\phi_{h}$ solves 3.6 with $\mathbf{f}=\omega^{2} \rho \boldsymbol{\psi}$ and $\mathbf{g} \equiv \mathbf{0}$. Thus, an application of Theorem 4.6 yields

$$
\begin{aligned}
\left\|\phi_{h}\right\|_{1, h}+\omega \rho^{\frac{1}{2}}\left\|\phi_{h}\right\|_{L^{2}(\Omega)} & \leq \frac{C}{\rho^{\frac{1}{2}}} C_{\text {sta }}\left\|\omega^{2} \rho \boldsymbol{\psi}\right\|_{L^{2}(\Omega)} \\
& \leq C \omega^{2} \rho^{\frac{1}{2}} C_{\text {sta }}\|\boldsymbol{\psi}\|_{L^{2}(\Omega)} .
\end{aligned}
$$

An application of Theorem 5.4 immediately infers 5.14).

We are now ready to derive error estimates for our IP-DG method. The next theorem is a consequence of combining Theorem 5.4 and Lemma 5.5.

TheOREm 5.6. Let $\mathbf{u} \in \mathbf{H}^{2}(\Omega)$ solve $(1.1)-\sqrt{1.2}$ and $\mathbf{u}_{h}$ be its IP-DG approximation. Then $\mathbf{u}-\mathbf{u}_{h}$ satisfies the following estimates:

$$
\begin{aligned}
& \left\|\mathbf{u}-\mathbf{u}_{h}\right\|_{1, h}+\omega \rho^{\frac{1}{2}}\left\|\mathbf{u}-\mathbf{u}_{h}\right\|_{L^{2}(\Omega)} \\
& \leq C \xi^{2} h\left(\xi+\gamma_{1}+\omega h\right)\left(\omega^{\alpha}+\frac{1}{\omega^{2}}\right)\left(\|\mathbf{f}\|_{L^{2}(\Omega)}+\|\mathbf{g}\|_{L^{2}(\Gamma)}\right) \\
& +C \xi^{2} \omega h^{2}\left(1+\omega C_{\text {sta }}\right)\left(\xi+\gamma_{1}+\omega h\right)\left(\omega^{\alpha}+\frac{1}{\omega^{2}}\right)\|\mathbf{f}\|_{L^{2}(\Omega)}, \\
& \left\|\mathbf{u}-\mathbf{u}_{h}\right\|_{L^{2}(\Omega)} \\
& \leq C \xi^{2} h^{2}\left(1+\omega C_{\text {sta }}\right)\left(\xi+\gamma_{1}+\omega h\right)\left(\omega^{\alpha}+\frac{1}{\omega^{2}}\right)\left(\|\mathbf{f}\|_{L^{2}(\Omega)}+\|\mathbf{g}\|_{L^{2}(\partial \Omega)}\right),
\end{aligned}
$$

where $C_{\mathrm{sta}}$ and $\xi$ are defined in 4.10), and $C$ is a positive constant independent of $\omega, h, \gamma_{0}, \gamma_{1}$.

Proof. Recall that $\mathbf{e}_{h}=\mathbf{u}-\mathbf{u}_{h}=\boldsymbol{\psi}+\boldsymbol{\phi}_{h}$ and the desired estimates for $\boldsymbol{\psi}$ and $\boldsymbol{\phi}_{h}$ have already been established in Theorem 5.4 and Lemma 5.5, respectively. These 
estimates are combined in the following steps to obtain (5.15):

$$
\begin{aligned}
&\left\|\mathbf{e}_{h}\right\|_{1, h}+\omega \rho^{\frac{1}{2}}\left\|\mathbf{e}_{h}\right\|_{L^{2}(\Omega)} \\
& \leq \| \boldsymbol{\psi}\left\|_{1, h}+\omega \rho^{\frac{1}{2}}\right\| \boldsymbol{\psi}\left\|_{L^{2}(\Omega)}+\right\| \boldsymbol{\phi}_{h}\left\|_{1, h}+\omega \rho^{\frac{1}{2}}\right\| \boldsymbol{\phi}_{h} \|_{L^{2}(\Omega)} \\
& \leq C \xi^{2} h\left(\xi+\gamma_{1}+\omega h\right)^{\frac{1}{2}}\left(\omega^{\alpha}+\frac{1}{\omega^{2}}\right)^{\frac{1}{2}}\left(\|\mathbf{f}\|_{L^{2}(\Omega)}+\|\mathbf{g}\|_{L^{2}(\Gamma)}\right) \\
&+C \xi^{2} \omega h^{2}\left(\xi+\gamma_{1}+\omega h\right)\left(\omega^{\alpha}+\frac{1}{\omega^{2}}\right)\left(\|\mathbf{f}\|_{L^{2}(\Omega)}+\|\mathbf{g}\|_{L^{2}(\Gamma)}\right) \\
&+C \xi^{2} \omega^{2} h^{2} C_{\text {sta }}\left(\xi+\gamma_{1}+\omega h\right)\left(\omega^{\alpha}+\frac{1}{\omega^{2}}\right)\left(\|\mathbf{f}\|_{L^{2}(\Omega)}+\|\mathbf{g}\|_{L^{2}(\Gamma)}\right) \\
& \leq C \xi^{2} h\left(\xi+\gamma_{1}+\omega h\right)\left(\omega^{\alpha}+\frac{1}{\omega^{2}}\right)\left(\|\mathbf{f}\|_{L^{2}(\Omega)}+\|\mathbf{g}\|_{L^{2}(\Gamma)}\right) \\
& \quad+C \xi^{2} \omega h^{2}\left(1+\omega C_{\text {sta }}\right)\left(\xi+\gamma_{1}+\omega h\right)\left(\omega^{\alpha}+\frac{1}{\omega^{2}}\right)\left(\|\mathbf{f}\|_{L^{2}(\Omega)}+\|\mathbf{g}\|_{L^{2}(\Gamma)}\right) .
\end{aligned}
$$

Similarly, 5.16 is obtained by combining Theorem 5.4 and Lemma 5.5. $\square$

REMARK 5.7. (a) The above estimates are sub-optimal in general due to the dependence of $C_{\mathrm{sta}}$ on the mesh size $h$. However, when h belongs to the pre-asymptotic mesh regime (i.e. when $\omega^{\beta} h=O(1)$ for $1 \leq \beta<1+\alpha$ ), $C_{\text {sta }}$ can be bounded by a constant which is independent of $h$ as explained in Remark 4.7. Therefore, the above error estimates become optimal (in $h$ ) in the pre-asymptotic mesh regime.

(b) Estimates that are optimal in $h$ in the asymptotic mesh regime can be found in [13].

6. Numerical experiments. In this section, we present some numerical tests to demonstrate key features of the proposed IP-DG method. In all our tests we choose $\Omega=(-0.5,0.5)^{2} \subset \mathbb{R}^{2}$ (i.e. the unit square in $\mathbb{R}^{2}$ centered at the origin), along with the material constants $\rho=\mu=\lambda=1$, and penalty constants $\gamma_{0}=10$ and $\gamma_{1}=0.1$. For the sake of testing the exact error, $\mathbf{f}$ and $\mathbf{g}$ are chosen so that the exact solution to the elastic Helmholtz problem is $\mathbf{u}=\frac{1}{\omega^{2} r}\left[e^{\mathbf{i} \omega r}-1, e^{-\mathbf{i} \omega r}-1\right]^{T}$, where $r=\|\mathbf{x}\|$ denotes the Euclidean length of $\mathbf{x}$. This simple problem along with the subsequent numerical tests are chosen to mirror those in 9] for the scalar Helmholtz problem. Some sample plots are given in Figures 6.2 and 6.3. These plots demonstrate how well the proposed IP-DG method can capture the wave with large frequency when using a relatively coarse mesh.

To partition the domain $\Omega$, a uniform triangulation $\mathcal{T}_{h}$ is used. For a positive integer $n$, define $\mathcal{T}_{1 / n}$ to be a triangulation of $2 n^{2}$ congruent isosceles triangles with side lengths $1 / n, 1 / n$, and $\sqrt{2} / n$. Figure 6.1 shows the triangulation $\mathcal{T}_{1 / 10}$.

The numerical tests in this section intend to demonstrate the following:

- absolute stability of the proposed IP-DG method,

- error of the proposed IP-DG solution,

- pollution effect on the error when $\omega h=O(1)$,

- absence of the pollution effect when $\omega^{3} h^{2}=O(1)$,

- performance comparison between standard FE and the proposed IP-DG method on the test problem.

6.1. Stability. In this subsection, the stability of both the proposed IP-DG method and the $P_{1}$-conforming finite element method will be discussed. Let $\mathbf{u}_{h}^{F E M}$ denote the $P_{1}$-conforming finite element approximation of $\mathbf{u}$. Recall that the proposed IP-DG approximation is unconditionally stable, i.e. it is stable for all $\omega, h, \gamma_{0}, \gamma_{1}>0$. 


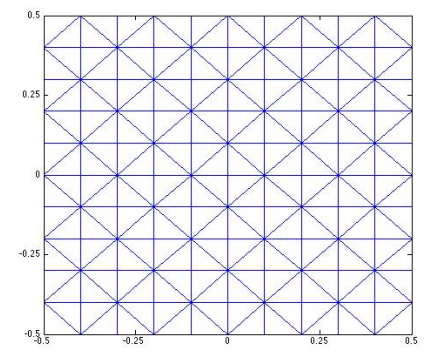

FIG. 6.1. The triangulation $\mathcal{T}_{1 / 10}$.
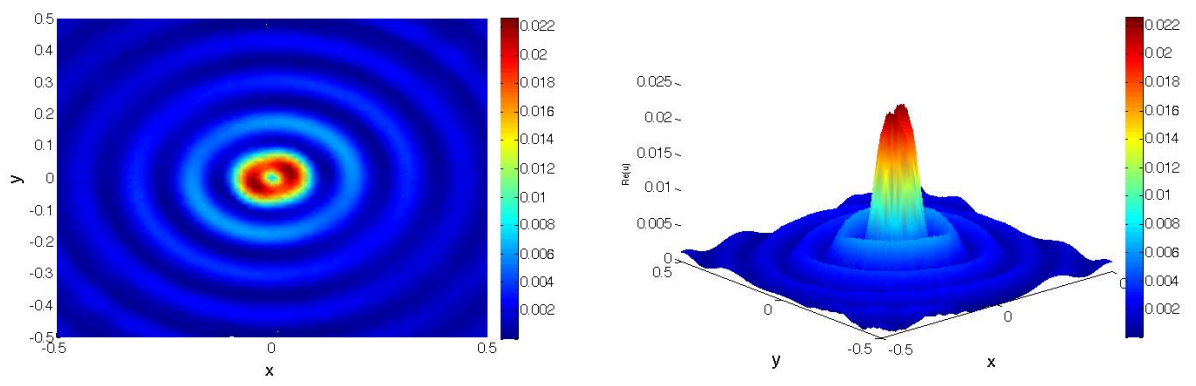

Fig. 6.2. Plot of $\left\|\operatorname{Re}\left(\mathbf{u}_{h}\right)\right\|_{L^{2}(\Omega)}$ for $\omega=50$ and $h=1 / 70$. Both a top down view (left) and a side view (right) are shown.

This has yet been established for the $P_{1}$-conforming finite element approximation. In fact, the stability of the $P_{1}$-conforming finite element approximation is only known to hold when $h$ satisfies $\omega^{2} h \leq C$.

Figure 6.4 plots both $\left\|\mathbf{u}_{h}\right\|_{1, h}$ and $\left\|\mathbf{u}^{F E M}\right\|_{1, h}$ for $h=0.05,0.01$ and $\omega=1,2, \cdots$, 200. We observe that $\left\|\mathbf{u}_{h}\right\|_{1, h}$ decreases in a smooth fashion as $\omega$ increases. This smooth behavior of $\left\|\mathbf{u}_{h}\right\|_{1, h}$ is indicative of the absolute stability of the IP-DG approximation. On the other hand, we observe oscillations in $\left\|\mathbf{u}_{h}^{F E M}\right\|_{1, h}$ that occur when we vary $\omega$. This oscillation is indicative of the instability of the $P_{1}$-conforming finite element method when $h$ is too large.

6.2. Error. In this subsection, the optimal order of convergence for the proposed IP-DG method will be demonstrated. The pollution effect will also be examined. From Theorems 5.6 and the error analysis for the asymptotic mesh regime carried out in 13 . (c.f. Remark 5.7) we expect the error in $\|\cdot\|_{1, h}$ to decrease at an optimal order in both the pre-asymptotic and asymptotic mesh regimes. In other words, $\left\|\mathbf{u}-\mathbf{u}_{h}\right\|_{1, h}=O(h)$ is expected. Figure 6.5 is a $\log -\log$ plot of the relative error $\left\|\mathbf{u}-\mathbf{u}_{h}\right\|_{1, h} /\|\mathbf{u}\|_{1, h}$ against the value $1 / h$ for frequencies $\omega=5,10,20,30$. From this plot, it is observed that the relative error decreases at the same rate as $h$, thus displaying the optimal order of convergence in the relative semi-norm. Also displayed in Figure 6.5 is the error when $\omega$ varies according to the constraint $\omega h=0.25$. From this figure it is observed that the error increases as $\omega$ increases under this constraint. This is due to the pollution effect on the error for the elastic Helmholtz problem.

The pollution effect for Helmholtz-type problems is characterized by the increase in error as $\omega$ is increased under the constraint $\omega h=O(1)$. This effect is intrinsic to 

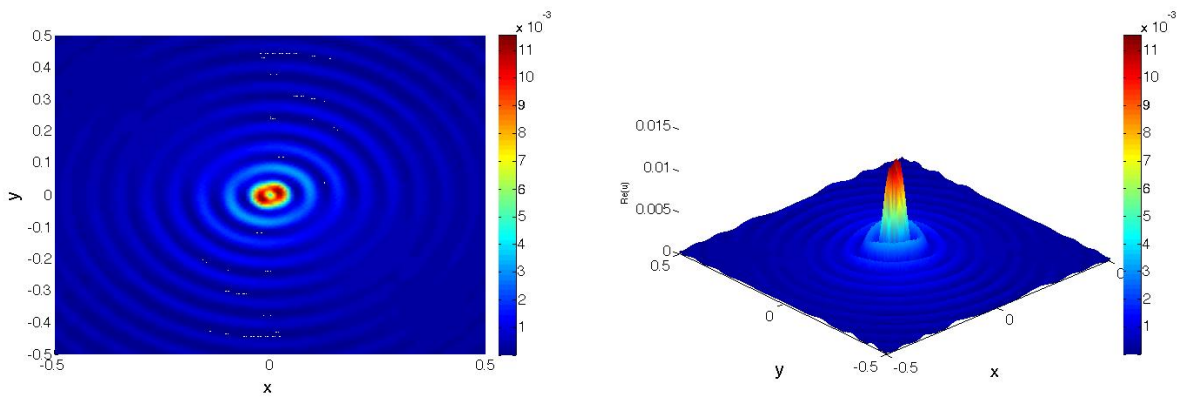

Fig. 6.3. Plot of $\left\|\operatorname{Re}\left(\mathbf{u}_{h}\right)\right\|_{L^{2}(\Omega)}$ for $\omega=100$ and $h=1 / 120$. Both a top down view (left) and a side view (right) are shown.
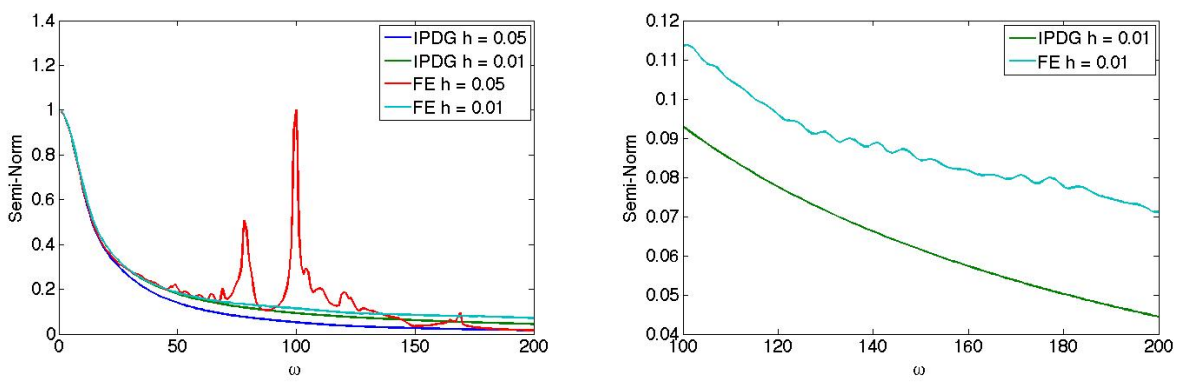

FIG. 6.4. Plots of $\left\|\mathbf{u}_{h}\right\|_{1, h}$ and $\left\|\mathbf{u}_{h}^{F E M}\right\|_{1, h}$.

Helmholtz-type problems (c.f. [12]). It is well-known that the pollution effect can be eliminated if $h$ is chosen to fulfill the stronger constraint $\omega^{3} h^{2}=O(1)$. In Figure 6.6 the relative error is plotted against $\omega$ as $h$ is chosen to satisfy different constraints. Under the constraints $\omega h=1$ and $\omega h=0.5$, the pollution effect is present and the relative error increases as $\omega$ is increased. On the other hand, when $\omega^{3} h^{2}=1$ is used to choose the the mesh size $h$, the pollution effect is eliminated.

6.3. Comparison between the IP-DG method and the FE method. In this subsection, we compare the performance of the proposed IP-DG method and the $P_{1}$-conforming finite element method. As stated previously, the proposed IP-DG method is unconditionally stable while the $P_{1}$-conforming finite element method is only shown to be stable when $h$ satisfies $\omega^{2} h=O(1)$. With this in mind, one can anticipate that in the case that the frequency $\omega$ is large, the IP-DG method should perform better.

In Figures 6.7 6.9, $\left\|\operatorname{Re}\left(\mathbf{u}_{h}\right)\right\|_{L^{2}(\Omega)}$ and $\left\|\operatorname{Re}\left(\mathbf{u}_{h}^{F E M}\right)\right\|_{L^{2}(\Omega)}$ are plotted for $\omega=100$ and $h=1 / 50,1 / 120,1 / 200$ on a cross-section over the line $y=x$. In addition, $\|\operatorname{Re}(\mathbf{u})\|_{L^{2}(\Omega)}$ is plotted to measure how well the respective approximations capture the true solution. In Figure 6.7, it is observed that $\mathbf{u}_{h}$ already captures the phase of $\mathbf{u}$ with $h=1 / 50$ while not fully capturing the large changes in magnitude. On the other hand, for $h=1 / 50, \mathbf{u}_{h}^{F E M}$ has spurious oscillations. In this case, $\mathbf{u}_{h}^{F E M}$ also fails to capture the changes in the magnitude of the wave. In Figure 6.8 we see that for $h=1 / 120, \mathbf{u}_{h}$ captures the phase and changes in magnitude of the wave very well while $\mathbf{u}_{h}^{F E M}$ still displays spurious oscillations. In Figure 6.9, we see for $h=1 / 200$, both methods capture the wave well. However, the IP-DG method cap- 


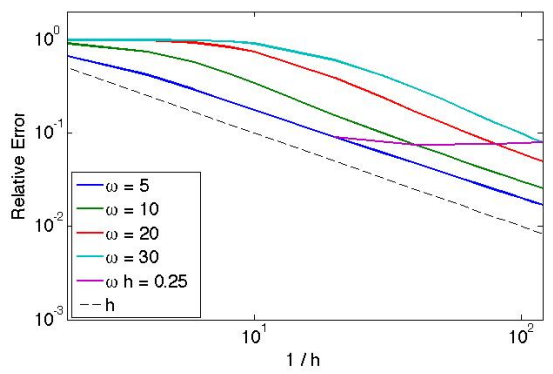

FIG. 6.5. Log-log plot of the relative error for the IP-DG approximation measured in the broken $H^{1}$-seminorm for different values of $\omega$.
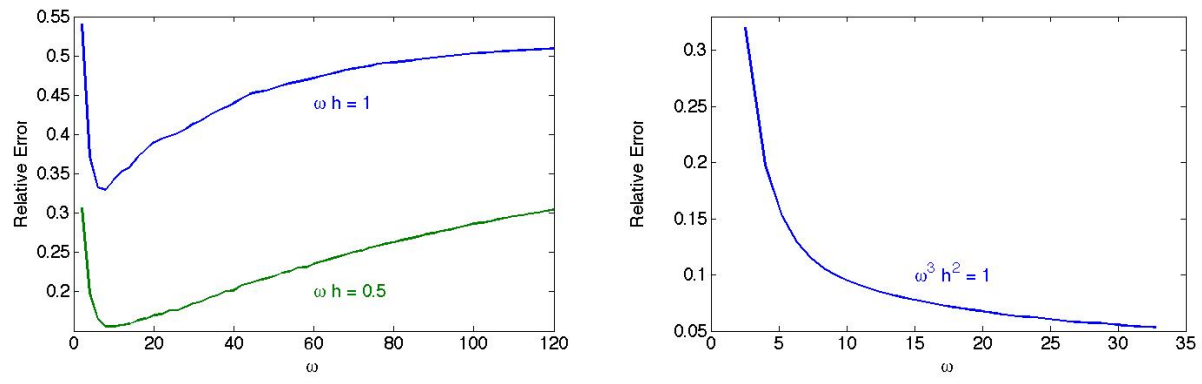

FIG. 6.6. Relative error of the IP-DG approximations measured in the broken $H^{1}$ seminorm computed for different pairs of $\omega$ and $h$ combinations.

tures the wave slightly better. These examples demonstrate that the IP-DG method approximates high frequency waves better than the standard finite element method does, in particular, on a coarse mesh. This is of great importance when memory is limited or one wishes to employ a multi-level solver such as multigrid or multi-level Schwarz space/domain decomposition methods.
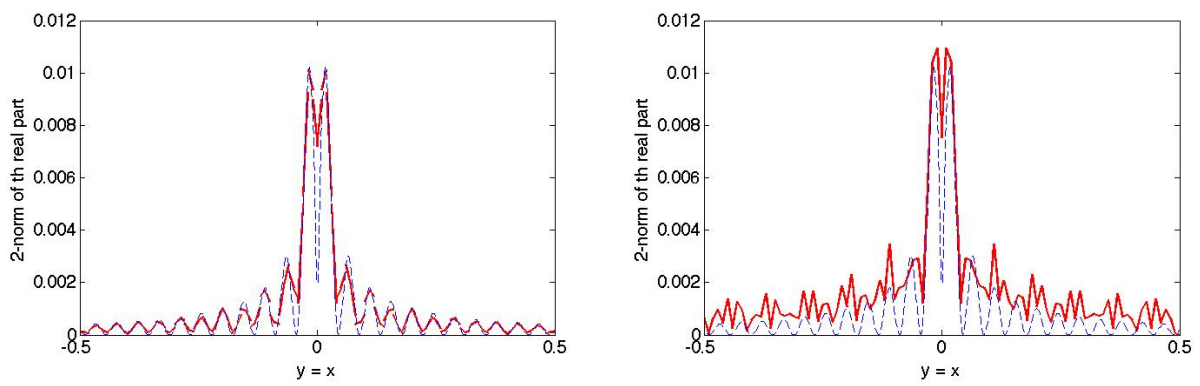

FIG. 6.7. The left plot is of $\left\|\operatorname{Re}\left(\mathbf{u}_{h}\right)\right\|_{L^{2}(\Omega)}$ (solid red line) vs. $\|\operatorname{Re}(\mathbf{u})\|_{L^{2}(\Omega)}$ (dashed blue line) for $h=1 / 50$. The right plot is of $\left\|\operatorname{Re}\left(\mathbf{u}_{h}^{F E M}\right)\right\|_{L^{2}(\Omega)}$ (solid red line) vs. $\|\operatorname{Re}(\mathbf{u})\|_{L^{2}(\Omega)}$ (dashed blue line) for $h=1 / 50$. 

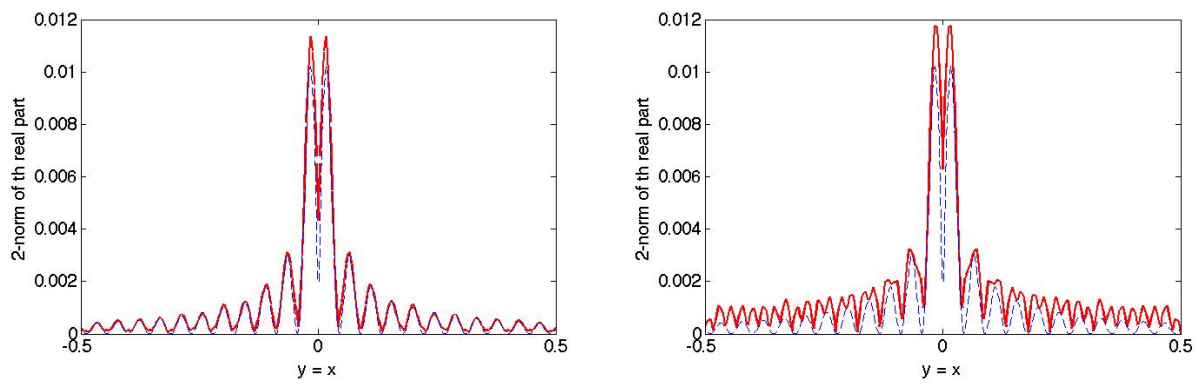

FIG. 6.8. The left plot is of $\left\|\operatorname{Re}\left(\mathbf{u}_{h}\right)\right\|_{L^{2}(\Omega)}$ (solid red line) vs. $\|\operatorname{Re}(\mathbf{u})\|_{L^{2}(\Omega)}$ (dashed blue line) for $h=1 / 120$. The right plot is of $\left\|\operatorname{Re}\left(\mathbf{u}_{h}^{F E M}\right)\right\|_{L^{2}(\Omega)}$ (solid red line) vs. $\|\operatorname{Re}(\mathbf{u})\|_{L^{2}(\Omega)}$ (dashed blue line) for $h=1 / 120$.
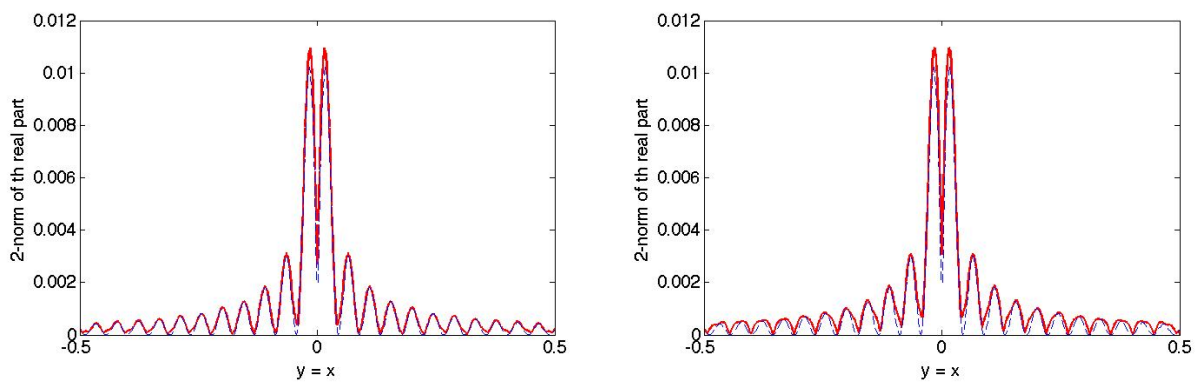

FiG. 6.9. The left plot is of $\left\|\operatorname{Re}\left(\mathbf{u}_{h}\right)\right\|_{L^{2}(\Omega)}$ (solid red line) vs. $\|\operatorname{Re}(\mathbf{u})\|_{L^{2}(\Omega)}$ (dashed blue line) for $h=1 / 200$. The right plot is of $\left\|\operatorname{Re}\left(\mathbf{u}_{h}^{F E M}\right)\right\|_{L^{2}(\Omega)}$ (solid red line) vs. $\|\operatorname{Re}(\mathbf{u})\|_{L^{2}(\Omega)}$ (dashed blue line) for $h=1 / 200$.

[1] A. K. Aziz and R. B. Kellogg. A scattering problem for the Helmholtz equation. Advances in computer methods for partial differential equations, III (Proc. Third IMACS Internat. Sympos., Lehigh Univ., Bethlehem, PA, 1979), pgs. 93-05. IMACS, New Brunswick, NJ, 1979.

[2] S. Brenner and R. Scott. The Mathematical Theory of Finite Element Methods. SpringerVerlag, New York, 2008.

[3] P.G. Ciarlet. The Finite Element Method for Elliptic Problems. North-Holland, Amsterdam, 1978.

[4] P. Cummings. Analysis of Finite Element Based Numerical Methods for Acoustic Waves, Elastic Waves and Fluid-Solid Interactions in the Frequency Domain. PhD thesis, The University of Tennessee, 2001.

[5] P. Cummings and X. Feng. Sharp Regularity Coefficient Estimates For Complex-Valued Acoustic and Elastic Helmholtz Equations. $M^{3} A S$, 16:139-160, 2006.

[6] J. Douglas Jr., J.E. Santos, D. Sheen, and L.S. Bennethum. Frequency domain treatment of one-dimensional scalar waves. $M^{3} A S, 3(2): 171-194,1993$.

[7] J. Douglas Jr., D. Sheen, and J.E. Santos. Approximation of scalar waves in the space-frequency domain. $M^{3} A S, 4(4): 509-531,1994$.

[8] B. Engquist and A. Majda. Radiation boundary conditions for acoustic and elastic wave calculations. Comm. Pure Appl. Math., 32(3):314-358, 1979.

[9] X. Feng and H. Wu. Discontinuous Galerkin methods for the Helmholtz equation with large wave numbers. SIAM J. Numer. Anal., 47:2872-2896, 2009.

[10] X. Feng and H. Wu. $h p$-Discontinuous Galerkin methods for the Helmholtz equation with large wave numbers. Math. Comp., 80:1997-2024, 2011.

[11] X. Feng and H. Wu. An absolutely stable discontinuous Galerkin method for the indefinite time-harmonic Maxwell equations with large wave number. SIAM J. Numer. Anal., in print, also downloadable at http://arxiv.org/pdf/1210.5837v2.pdf 
[12] F. Ihlenburg and I. Babǔska. Finite element solution of the Helmholtz equation with high wave number. The $h$-version of FEM. Comput. Math. Appl., 30(9):9-37, 1995.

[13] C. Lorton. Numerical methods and algorithms for high frequency wave scattering problems in homogeneous and random media. PhD thesis, The University of Tennessee, 2014.

[14] B. Riviere, S. Shaw, M.F. Wheeler and J.R. Whiteman. Discontinuous Galerkin finite element methods for linear elasticity and quasistatic linear viscoelasticity. Numer. Math., 95(2):347$376,2003$.

[15] A.H. Schatz. An observation concerning Ritz-Galerkin methods with indefinite bilinear forms. Math. Comp., 28:959-962, 1974.

[16] H. Wu. Pre-asymptotic error analysis of CIP-FEM and FEM for the Helmholtz equation with high wave number. Part I: linear version. IMA J. Numer. Anal., 2013 